\title{
Long-term variability of AGN at hard X-rays *
}

\author{
S. Soldi ${ }^{1}$, V. Beckmann ${ }^{2}$, W. H. Baumgartner ${ }^{3,4}$, G. Ponti ${ }^{5}$, C. R. Shrader ${ }^{3,6}$, \\ P. Lubiński ${ }^{7}$, H. A. Krimm ${ }^{3,6}$, F. Mattana ${ }^{2}$, and J. Tueller ${ }^{3}$ \\ 1 APC, Université Paris Diderot, CNRS/IN2P3, CEA/Irfu, Observatoire de Paris, Sorbonne Paris Cité, \\ 10 rue Alice Domon et Léonie Duquet, 75205 Paris Cedex 13, France \\ e-mail: soldi@apc.univ-paris7.fr \\ 2 François Arago Centre, APC, Université Paris Diderot, CNRS/IN2P3, CEA/Irfu, Observatoire de Paris, Sorbonne Paris Cité, \\ 13 rue Watt, 75013 Paris, France \\ 3 NASA Goddard Space Flight Center, Greenbelt MD 20771, USA \\ 4 Joint Center for Astrophysics, University of Maryland Baltimore County, Baltimore MD 21250, USA \\ 5 Max-Planck-Institut für extraterrestrische Physik, Giessenbachstrasse 1, 85748 Garching bei München, Germany \\ ${ }^{6}$ CRESST, Universities Space Research Association and NASA GSFC, Greenbelt MD 20771, USA \\ 7 Institute of Physics, University of Zielona Góra, Licealna 9, 65-417 Zielona Góra, Poland
}

Received 12 September 2013 / Accepted 15 November 2013

\section{ABSTRACT}

\begin{abstract}
Aims. Variability at all observed wavelengths is a distinctive property of active galactic nuclei (AGN). Hard X-rays provide us with a view of the innermost regions of AGN, mostly unbiased by absorption along the line of sight. Characterizing the intrinsic hard X-ray variability of a large AGN sample and comparing it to the results obtained at lower X-ray energies can significantly contribute to our understanding of the mechanisms underlying the high-energy radiation.

Methods. Swift/BAT provides us with the unique opportunity to follow, on time scales of days to years and with regular sampling, the 14-195 keV emission of the largest AGN sample available up to date for this kind of investigation. As a continuation of an early work using the first 9 months of BAT data, we study the amplitude of the variations and their dependence on subclass and on energy, for a sample of 110 radio quiet and radio loud AGN selected from the BAT 58-month survey.

Results. About $80 \%$ of the AGN in the sample are found to exhibit significant variability on month-to-year time scales. In particular, radio loud sources are the most variable, and Seyfert 1.5-2 galaxies are slightly more variable than Seyfert 1, while absorbed and unabsorbed objects show similar timing properties. The amplitude of the variations and their energy dependence are incompatible with variability being driven at hard X-rays by changes in the absorption column density. In general, the variations in the 14-24 and 35-100 keV bands are correlated well, suggesting a common origin to the variability across the BAT energy band. However, radio quiet AGN display on average 10\% larger variations at $14-24 \mathrm{keV}$ than at 35-100 keV, and a softer-when-brighter behavior for most of the Seyfert galaxies with detectable spectral variability on a time scale of a month. In addition, sources with harder spectra are found to be more variable than softer ones, unlike what it is observed below $10 \mathrm{keV}$. These properties are generally consistent with a variable, in flux and shape, power law continuum, pivoting at energies $\gtrsim 50 \mathrm{keV}$, to which a constant reflection component is superposed. When the same time scales are considered, the timing properties of AGN at hard X-rays are comparable to those at lower energies, with at least some of the differences possibly ascribable to components contributing differently in the two energy domains (e.g., reflection, absorption).
\end{abstract}

Key words. galaxies: active - galaxies: Seyfert - X-rays: galaxies - surveys

\section{Introduction}

Besides mapping the innermost regions of active galactic nuclei (AGN), the hardest X-rays $\gtrsim 15 \mathrm{keV}$ present the further advantage of not being affected by absorption, provided that the hydrogen column density $N_{\mathrm{H}}$ along the line of sight is lower than a few $10^{23} \mathrm{~cm}^{-2}$. As a result, hard X-rays can be effectively used to study the intrinsic properties of AGN emission, testing the validity of unification models that in first approximation explain the differences between the different AGN classes as a function of the viewing angle (e.g., Beckmann \& Shrader 2012). In radio quiet AGN, the hard X-ray emission is postulated to originate in a two-phase medium, where the soft disk photons are Comptonized by electrons in a hot plasma above the disk (Haardt \& Maraschi 1993). In radio loud objects, an additional contribution from inverse Compton scattering from relativistic electrons

* Table 2 is available in electronic form at http://www. aanda.org in the jet, as well as synchrotron radiation, can dominate the $\mathrm{X}$-ray output.

Moreover, AGN are known to exhibit variability at all observed frequencies, whose study can provide important information about the physics, the structure, and the dynamics of the emitting source. In the softer X-ray domain $(\$ 10 \mathrm{keV})$, extensive variability studies have been carried out, especially in the recent years thanks to the numerous monitoring campaigns with RXTE and XMM-Newton, covering time scales from hours to years. Many important results have been obtained with these observations, such as the complex correlation of the X-rays with the emission at other wavelengths (e.g., McHardy et al. 2007; Soldi et al. 2008; Bell et al. 2011; Mehdipour et al. 2011; Chatterjee et al. 2011), in particular, in the optical-UV band (e.g., Shemmer et al. 2003; Uttley 2005; Arévalo et al. 2008b; Breedt et al. 2010); the study of X-ray lag spectra (e.g., Arévalo et al. 2008a; Zoghbi et al. 2010; De Marco et al. 2013); the correlations 
between variability properties and black hole mass (e.g., Czerny et al. 2001; Lu \& Yu 2001; Uttley et al. 2002; Papadakis 2004) and accretion rate (McHardy et al. 2006; Körding et al. 2007). See McHardy (2010) for a review of X-ray variability and its scaling from stellar to super-massive black holes. On the other hand, the large majority of the hard X-ray variability studies performed in the last years focused on the spectral variability of AGN at different flux levels for single bright sources or for small samples of selected objects, thanks to pointed observations of the BeppoSAX (e.g., Petrucci et al. 2000; Nicastro et al. 2000; de Rosa et al. 2007), INTEGRAL (e.g., Pian et al. 2006, 2011; Beckmann et al. 2008; Lubiński et al. 2010; Soldi et al. 2011a; Petrucci et al. 2013) and Suzaku (e.g., Reeves et al. 2007; Itoh et al. 2008; Terashima et al. 2009; Fukazawa et al. 2011; Reis et al. 2012) satellites. These X-ray studies suggest different origins for the observed variability, such as changes in the amount of absorption or of its ionization, fluctuations in the seed photon flux, intrinsic modifications of the corona properties or geometry (or of the jet parameters, in case of radio loud AGN), or a variable contribution of different spectral components, with at least some of these scenarios driven by accretion rate variations.

However, with the only exception being the CGRO/BATSE instrument, detecting only a handful of AGN (Harmon et al. 2004), long-term hard X-ray monitoring has not been possible until recent years, because of the observing strategy of the hard X-ray satellites and the limited size of the field of view of their instruments. Furthermore, latest generation and future hard-X-ray telescopes such as NuSTAR will have small fields of view and relatively inflexible scheduling capabilities, so future studies may tend to be limited to either short time scales or in the number of observed objects. Since November 2004, the Burst Alert Telescope (BAT, Barthelmy et al. 2005) instrument on board the Swift satellite (Gehrels et al. 2004) has been observing the sky in the $14-195 \mathrm{keV}$ energy range. Thanks to its large field of view of $\sim 1.4 \mathrm{sr}$ and to Swift's observing strategy, the BAT has been monitoring a large number of hard X-ray sources (Cusumano et al. 2010; Baumgartner et al. 2013), providing a long-term and sufficiently sampled data set for the first time. Swift/BAT light curves on different time scales and energy bands and extracted with different methods are provided through the hard X-ray transient monitoring pages ${ }^{1}$ (Krimm et al. 2013) and for the BAT 58-month hard X-ray survey ${ }^{2}$ that includes more than 1000 objects, about $60 \%$ of which are AGN. The latter has been recently updated to cover 70 months of observations (Baumgartner et al. 2013).

A first study of the variability of hard X-ray-selected AGN using BAT data has been presented by Beckmann et al. (2007a). It includes 44 AGN detected with high significance over the first nine months of BAT observations. The variability study in the 14-195 keV band was performed using a maximum likelihood estimator and the structure function analysis. Among the main results of this work, Seyfert 2/obscured objects were found to be more variable than Seyfert 1/unobscured ones, and an anticorrelation was detected between variability and luminosity.

Preliminary studies of the five years BAT observations but with limited numbers of AGN have been presented by Soldi et al. (2010), Ricci et al. (2011a), and Caballero-Garcia et al. (2012). In particular this last study focused on five bright AGN, detecting spectral variability in three of them, which is consistent with

\footnotetext{
1 http://swift.gsfc.nasa.gov/results/transients/

2 http://swift.gsfc.nasa.gov/results/bs58mon/index.php
}

the electron plasma temperature decreasing with increasing flux, within the Comptonization scenario.

Shimizu \& Mushotzky (2013, hereafter SM13) have calculated the power density spectra (PDS) of 30 AGN for the first time in hard X-rays (all these objects are also included in our study), using BAT $14-150 \mathrm{keV}$ data covering time scales from one week to a few years ( $\left.v_{\mathrm{PDS}} \sim 10^{-8}-10^{-6} \mathrm{~Hz}\right)$. All but one PDS were found to be well fitted by a simple power law with slope $\alpha \sim-0.8$, similar to the PDS measured in the $2-10 \mathrm{keV}$ domain at time frequencies lower than a break frequency $1 / T_{\mathrm{B}}$. In fact, the PDS of AGN in the $2-10 \mathrm{keV}$ domain has a characteristic power law shape with index $\alpha_{\mathrm{PDS}}=-1$ for long time scales, steepening to $\alpha_{\mathrm{PDS}}=-2$ for times scales shorter than a source-specific break time $T_{\mathrm{B}}$ (Uttley 2005; González-Martín \& Vaughan 2012), which is found to scale with black hole mass and accretion rate following the relation described by McHardy et al. (2006). The lack of correlations between variability and luminosity, and between variability and black hole mass, in the sample presented by Shimizu \& Mushotzky (2013) has, therefore, been ascribed to the long time scales probed by this study. No decisive evidence was found to determine whether AGN longterm variability is energy dependent.

We present here our variability analysis of the largest AGN sample with a sufficient hard X-ray monitoring to perform this kind of study. Our analysis provides an orthogonal approach to the one presented by SM13, since we consider the frequencyindependent variations of the full long-term light curves, and therefore we are able to extend our study to a much larger AGN sample, while for the PDS analysis, a high signal-to-noise ratio $(\mathrm{S} / \mathrm{N})$ is required, thus limiting the use of this technique to the 30 objects already presented by SM13. Furthermore, we are also able to investigate the dependence of variability on energy in the hard X-ray band.

A description of the AGN light curves in the BAT 58-month survey is presented in Sect. 2, along with the estimate of the systematic uncertainties. In Sect. 3 the maximum likelihood estimator for variability is introduced and the selected sample of 110 AGN described. The correlation analysis between variability and the AGN fundamental parameters is detailed in Sect. 4, while Sect. 5 presents the study of spectral variability within the BAT energy range. We discuss our results (Sect. 6) with particular emphasis on the comparison between hard X-ray and soft $\mathrm{X}$-ray variability, since this can provide important insight into the mechanisms responsible for the observed emission across the high-energy spectrum. A summary of our conclusions is given in Sect. 7.

Considering the importance of the analyzed time scales in the results presented here, we generally refer to long time scales (and low time frequencies) for variability studies considered to sample the PDS above the break $T_{\mathrm{B}}$, and to short time scales (and high time frequencies) below the break. In the following we use a $\Lambda$ CDM cosmology with $\Omega_{\mathrm{M}}=0.3, \Omega_{\Lambda}=0.7$, and $H_{0}=73 \mathrm{~km} \mathrm{~s}^{-1} \mathrm{Mpc}^{-1}$.

\section{Swift/BAT light curve analysis}

Among the 1092 sources detected in the BAT 58-month survey, 625 are AGN. We exclude from this list the six pairs of AGN whose sky positions are too close to safely avoid contamination of the light curves, i.e., objects with BAT coordinates within 2 arcmin of each other. For these 613 AGN, we retrieved the publicly available light curves covering the time from the beginning of the mission up to May 2010, therefore 
including up to 66 months of data ${ }^{3}$. The light curves have been extracted from the snapshot (i.e. a single Swift pointing lasting about $20 \mathrm{~min}$ ) images corrected for off-axis effects. The images were created in eight energy bands, i.e., 14-20, 20-24, 24-35, 35-50, 50-75, 75-100, 100-150, and 150-195 keV. To convert count rates in the total $14-195 \mathrm{keV}$ band to fluxes, we assume a Crab-like spectrum $(\Gamma=2.15$; Tueller et al. 2010) with a flux of $F_{\mathrm{Crab}, 14-195 \mathrm{keV}}=2.44 \times 10^{-8} \mathrm{erg} \mathrm{cm}^{-2} \mathrm{~s}^{-1}$ corresponding to a count rate of $x_{\mathrm{Crab}, 14-195 \mathrm{keV}}=0.0418$ counts s$^{-1}$ detector $^{-1}$ (average from the Crab snapshot light curve). The complete description of the BAT data analysis and light curve extraction is provided in Tueller et al. (2010) and Baumgartner et al. (2013).

The reference light curves used for our analysis are obtained from the combined 14-195 keV light curves first rebinned to 30 days and then filtered to exclude points with exposure time shorter than one day and data points with error bars larger than $\Delta F_{\text {cut }, 30 \text { days }}=1.7 \mathrm{mCrab}$. To derive this value, the histogram of the flux uncertainties for all light curves was built in logarithmic space, and the histogram peak and minimum identified. The value $\Delta F_{\text {cut, } 30 \text { days }}=2 \times \Delta F_{\text {peak }}-\Delta F_{\text {min }}$ (i.e., as far above the peak of the distribution as the minimum is below) is considered to mark the beginning of the high-value tail of the distribution of flux uncertainties and is, therefore, used as the cut-off value, $\Delta F_{\text {cut }, 30 \text { days }}$.

\subsection{Light curve systematic errors}

Residual systematic uncertainties can affect the light curves and need to be taken into account before a variability analysis can be performed. Among them, the most important contributions come from a component $\sigma_{\text {sys, } \mathrm{A}}=s y s_{\mathrm{A}} \times F$, which is proportional to the source flux, a component $\sigma_{\text {sys, B }}=$ sys $_{\mathrm{B}} \times \sigma_{\text {stat }}$ that can be derived from empty sky positions and a term $\sigma_{\text {sys, } C}=\operatorname{sys}_{\mathrm{C}} \times \sigma_{\text {stat }}$ due to summing up energy and time bins that are somehow correlated. The term $\sigma_{\text {sys, A }}$ is estimated using the Crab light curve under the assumption that it is constant. Fitting the Crab orbital light curve with a constant, a $\sigma_{\text {sys, }}=0.06 \times F$ systematic error needs to be added to obtain a reduced $\chi^{2}$ equal to 1 . A further source of uncertainty is expected following the finding of longterm variability in the Crab light curve (up to $\sim 7 \%$ on a year time scale; Wilson-Hodge et al. 2011); therefore, the value of the sys $\mathrm{s}_{\mathrm{A}}$ term is likely to be overestimated here. Owing to the difficulty of disentangling the systematic component from the intrinsic Crab variability, we prefer to choose a conservative approach and to adopt the sys $_{\mathrm{A}}=0.06$ value. In any case, even when reducing this term to 0.01 , the amplitude of the variations increases on average by only $2 \%$ to $3 \%$, largely within the estimated uncertainties on the variability estimator (see Sect. 3.1).

The component $\sigma_{\text {sys,B }}$ is estimated by constructing orbital light curves from blank sky positions and building the histogram of all the resulting significances. By selecting sky positions with no sources, a Gaussian distribution centered on zero for the significances is expected. Any significant deviation from a zero mean value or from a width equal to one would point towards systematic uncertainties. Indeed, the statistical errors need to be increased by $\sigma_{\text {sys,B }}=0.08 \times \sigma_{\text {stat }}$ in order to obtain a Gaussian fit to the histogram of significances with width equal to one.

Finally, from the comparison between the 14-195 keV monthly light curves extracted from the one-month integrated mosaic images and the monthly light curves obtained by

\footnotetext{
3 We note that even though 58 months of BAT data were used for source detection, the light curves used in this work extend beyond this data set, i.e. up to 66 months.
}

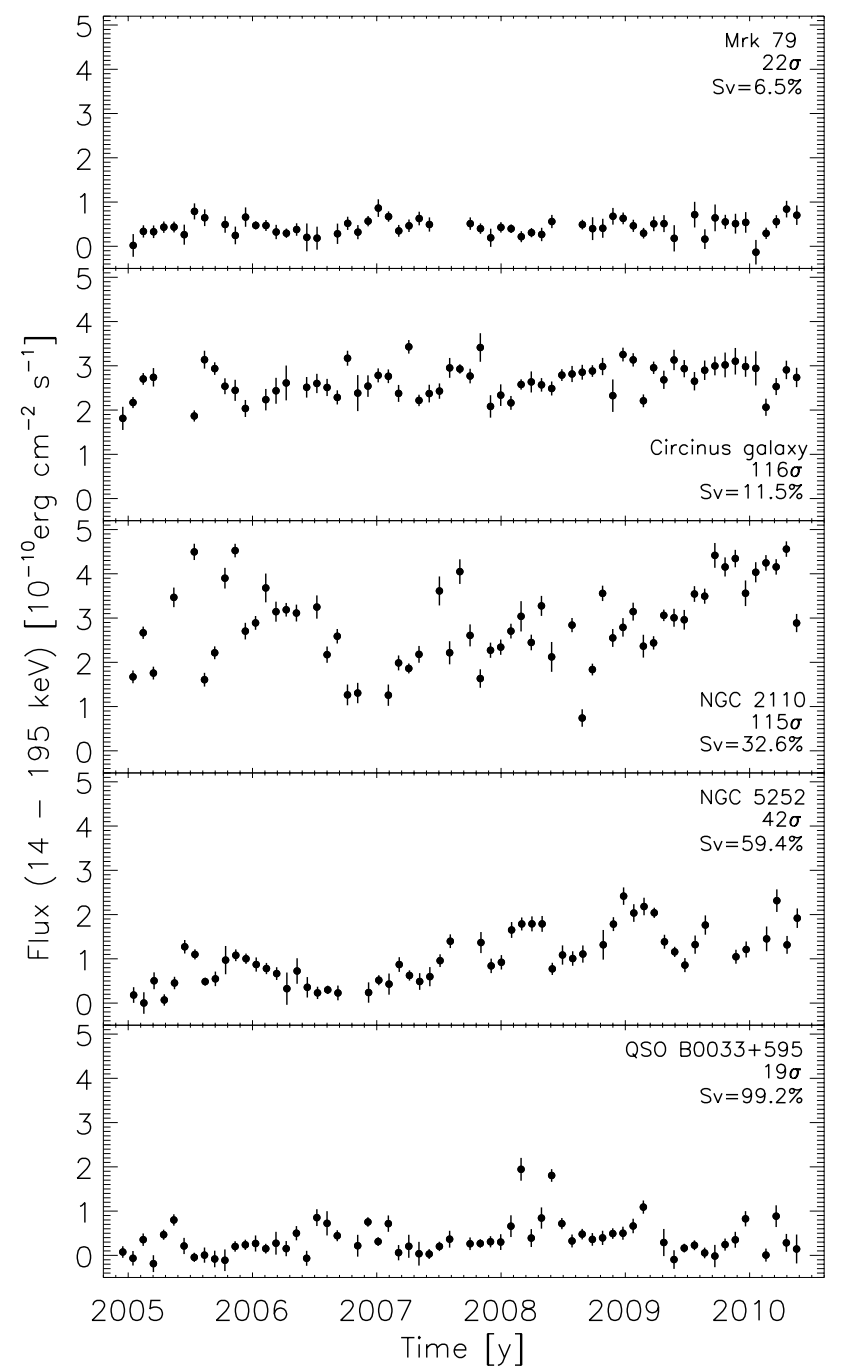

Fig. 1. Examples of 30-d binned, 14-195 keV BAT light curves for objects presenting different variability levels (increasing from top to bottom) and with different signal-to-noise ratios. The estimator $S_{V}$ represents the intrinsic variability of a light curve, renormalized to the average flux and in percentage (see Sect. 3.1).

rebinning the orbital light curves and summing up eight energy bands over the full $14-195 \mathrm{keV}$ range, we estimate that an additional statistical error contribution, $\sigma_{\text {sys, }}=0.08 \times \sigma_{\text {stat }}$, is needed to take the effects of correlated energy and time bins into account. The final statistical plus systematic error is given by $\sigma_{\text {tot }}=\sqrt{\left(\operatorname{sys}_{\mathrm{A}} \times F\right)^{2}+\left[\sigma_{\text {stat }}\left(1+\text { sys }_{\mathrm{B}}+\text { sys }_{\mathrm{C}}\right)\right]^{2}}$. Figure 1 presents five example light curves with different $\mathrm{S} / \mathrm{Ns}$ and variability.

\section{Hard X-ray variability properties}

As an initial estimate of the variability of our AGN sample, we fit the 30-day binned light curves of the 613 AGN with a constant function, and apply a $\chi^{2}$ test. The variability index $V$ is defined as $V=-\log \left(1-P_{\chi^{2}}\right)$, where $P_{\chi^{2}}$ is the null hypothesis probability to obtain such a $\chi^{2}$ if the source were constant. For $36 \%$ of the light curves in the sample the fit to a constant intensity source results in $V>1.3$ (i.e., $P_{\chi^{2}} \leq 5 \%$; Paolillo et al. 2004; Lanzuisi et al. 2013), indicating that these objects exhibit significant variability (Fig. 2). In particular, $34 \%$ of the Seyfert galaxies are found to be variable against the $44 \%$ of the radio loud 

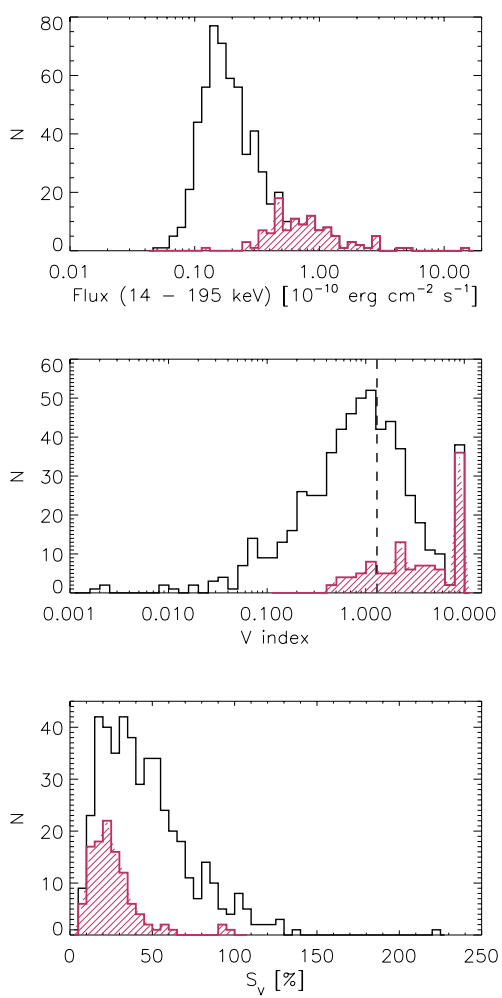

Fig. 2. Histograms of the time-averaged $14-195 \mathrm{keV}$ flux (top), of the variability index $V$ from the $\chi^{2}$ test (middle; the vertical dashed line indicates $V=1.3$ ), and of the variability amplitude estimator $S_{V}$ (bottom) for the total sample of 613 AGN (black) and for the selected sample of 110 AGN (red hatched). We assigned $V=10$ to those objects with $P_{\chi^{2}}<10^{-6} . S_{V}$ could be computed for 464 of the BAT-detected AGN.

population. Among Seyfert galaxies, about $41 \%$ of type- 2 objects show variability, contrary to only $29 \%$ of the type- 1 objects.

\subsection{Amplitude of the variations}

Several methods can be used to quantify the amplitude of the variations in a light curve, e.g. excess variance or fractional variability amplitude (Vaughan et al. 2003; Ponti et al. 2004; Soldi et al. 2008). Almaini et al. (2000) proposed a numerical approach, arguing that this is a more appropriate method in the presence of non-uniform measurement uncertainties, while recently Allevato et al. (2013) have shown that it provides results equivalent to the normalized excess variance. The Almaini method is based on a maximum-likelihood estimate of the $\sigma_{\mathrm{Q}}$ parameter, representing the variability of the light curve. In the case of constant measurement uncertainties $\left(\sigma_{\mathrm{i}}=\right.$ const. $)$, it reduces to the excess variance $\left(\sigma_{\mathrm{Q}}=\sigma_{\mathrm{XS}}\right)$. The $\sigma_{\mathrm{Q}}$ parameter is defined so as to satisfy the following equation (see Almaini et al. 2000; and Beckmann et al. 2007a, for more details):

$$
\sum_{i=1}^{N} \frac{\left[\left(x_{\mathrm{i}}-\bar{x}\right)^{2}-\left(\sigma_{\mathrm{i}}^{2}+\sigma_{\mathrm{Q}}^{2}\right)\right]}{\left(\sigma_{\mathrm{i}}^{2}+\sigma_{\mathrm{Q}}^{2}\right)^{2}}=0
$$

where $\bar{x}$ is the mean value of the light curve $x_{\mathrm{i}}$ with measurement uncertainties $\sigma_{\mathrm{i}}$. The maximum-likelihood estimator has also been successfully used in, say, Mateos et al. (2007) and in Chitnis et al. (2009). For continuity and comparison with Beckmann et al. (2007a), we choose to apply the Almaini method here. Unlike the original prescription, we use the weighted rather than the arithmetic mean to compute $\sigma_{\mathrm{Q}}$, because it provides more stable results in general, being less sensitive to the presence of outlier points. Nevertheless, we verified that equivalent results are obtained in both cases when analyzing the final AGN sample presented here.

To compare the variability of different objects, we renormalize $\sigma_{\mathrm{Q}}$ to the average flux $\langle F\rangle$ of the source, obtaining the $S_{V}=\sigma_{\mathrm{Q}} /\langle F\rangle \times 100 \%$ variability estimator (Fig. 2). Therefore, $S_{V}$ measures, in percentage units, the amplitude of the intrinsic variability of a light curve, corrected by measurement uncertainties and renormalized to the average flux. Unlike in Beckmann et al. (2007a), the systematic uncertainties deduced from the $\mathrm{Crab}$ and blank sky positions have already been included in the orbital light curves and no further correction to $S_{V}$ is therefore required.

In computing $S_{V}$, the uncertainty $\sigma_{\text {meas }}$ is determined with a bootstrap technique (Simpson \& Mayer-Hasselwander 1986). For each object, 100000 light curves are randomly drawn from the original one, with the same total number of points and allowing the same data point to be drawn more than once. The variability estimator $S_{V}$ is then computed for each simulated light curve and its frequency distribution built. The 15.9th and 84.1th percentiles of this distribution are taken as an estimate of the $1 \sigma$ confidence intervals (see also Vaughan et al. 2003). Owing to the stochastic nature of variability, a further source of uncertainty $\sigma_{\text {sampl }}$ is introduced by the uneven sampling of the light curves. This contribution can be understood as the dispersion of $S_{V}$ values measured on the same light curve on which different samplings are applied. We estimate this contribution with the bootstrap method, but this time using the initial, unfiltered light curves (i.e., all having regular 30-day sampling). For each object, 10000 light curves with $N$ points are randomly drawn from the original one, where $N$ is the number of points of the filtered light curve and where every data point cannot be drawn more than once. The 15.9th and 84.1th percentiles of the $S_{V}$ frequency distribution are taken as an estimate of the $1 \sigma$ confidence intervals. The final uncertainty on $S_{V}$ is obtained by combining in quadrature $\sigma_{\text {meas }}$ and $\sigma_{\text {sampl }}$.

\subsection{Sample selection}

The final sample of 110 AGN was selected based on the following two criteria: 1) the average value of the $\mathrm{S} / \mathrm{N}$ of the points in the light curve is larger than $2 ; 2) S_{V}$ can be measured for the given 30-day binned, filtered light curve, i.e., the uncertainties are smaller than the measurable intrinsic variability. The first criterion is essentially (but not exactly) equivalent to using a flux threshold of $F(14-195 \mathrm{keV})>3.8 \times 10^{-11} \mathrm{erg} \mathrm{cm}^{-2} \mathrm{~s}^{-1}$ or a $\mathrm{S} / \mathrm{N}$ over the full light curve larger than 15 , and it selects 115 sources (Fig. 2). The second criterion excludes only five additional sources. The filtering applied to the light curves (see Sect. 2) excludes no more than $22 \%$ ( $\sim 10 \%$ on average) of the data for the objects in the selected sample ${ }^{4}$. The resulting sample is composed of 88 Seyfert galaxies (36 Seyfert 1, 17 of intermediate type, 32 Seyfert 2, and 3 Narrow Line Seyfert 1), 9 radio galaxies and 13 blazars. These sources cover a range of 14-195 keV fluxes between $F=10^{-11}$ and $10^{-9} \mathrm{erg} \mathrm{cm}^{-2} \mathrm{~s}^{-1}$

4 The values of $S_{V}$ computed on the filtered and unfiltered light curves are compatible, with an average scatter of $\leq 2 \%$ for the selected sample, well within the estimated uncertainties on $S_{V}$. On the other hand, for the BAT AGN excluded from the analysis the scatter is on average 4 times larger, further justifying our choice to limit the analysis to a sample of light curves for which the variability measurement is not so strongly influenced by single data points. 
Table 1. Average properties of the BAT AGN in our variability sample, as a function of the AGN class and the amount of intrinsic absorption.

\begin{tabular}{lcccccc}
\hline \hline Type & $\langle z\rangle^{a}$ & $\begin{array}{c}\left\langle F_{14-195 \mathrm{keV}\rangle^{a}}\right. \\
{\left[10^{-11} \mathrm{erg} \mathrm{cm}^{-2} \mathrm{~s}^{-1}\right]}\end{array}$ & $\langle\Gamma\rangle^{a}$ & $\begin{array}{c}\left\langle S_{V}\right\rangle^{a} \\
{[\%]}\end{array}$ & $\begin{array}{c}\left\langle S_{\mathrm{V}, 14-24 \mathrm{keV}}\right\rangle^{b} \\
{[\%]}\end{array}$ & $\begin{array}{c}\left\langle S_{\mathrm{V}, 35-100 \mathrm{keV}}\right\rangle^{b} \\
{[\%]}\end{array}$ \\
\hline Sey 1 & $0.033(36)$ & $6.4 \pm 0.8$ & $2.01 \pm 0.02$ & $16.5 \pm 0.9$ & $21 \pm 2(15)$ & $13 \pm 2$ \\
Sey 1.5 & $0.017(17)$ & $10 \pm 2$ & $1.92 \pm 0.03$ & $24 \pm 1$ & $32 \pm 2(11)$ & $23 \pm 2$ \\
Sey 2 & $0.016(32)$ & $10 \pm 1$ & $1.86 \pm 0.03$ & $19.9 \pm 0.7$ & $28 \pm 1(22)$ & $16.2 \pm 0.9$ \\
NLS1 & $0.019(3)$ & $11 \pm 6$ & $2.2 \pm 0.1$ & $15 \pm 3$ & $24 \pm 4(2)$ & $9 \pm 4$ \\
\hline unabs Sey & $0.031(41)$ & $6.7 \pm 0.7$ & $2.00 \pm 0.02$ & $18.5 \pm 0.8$ & $25 \pm 2(23)$ & $17 \pm 2$ \\
abs Sey & $0.016(46)$ & $10 \pm 1$ & $1.90 \pm 0.03$ & $19.7 \pm 0.6$ & $28 \pm 1(26)$ & $16.4 \pm 0.8$ \\
CT Sey & $0.011(6)$ & $13 \pm 4$ & $1.8 \pm 0.1$ & $16 \pm 1$ & $37 \pm 6(4)$ & $9 \pm 1$ \\
\hline all Sey & $0.023(88)$ & $8.5 \pm 0.8$ & $1.95 \pm 0.02$ & $19.3 \pm 0.5$ & $26.9 \pm 0.9(50)$ & $16.7 \pm 0.7$ \\
radio galaxies & $0.033(9)$ & $22 \pm 12$ & $2.1 \pm 0.2$ & $24 \pm 1$ & $26 \pm 2(9)$ & $26 \pm 2$ \\
blazars/quasars & $0.855(13)$ & $7 \pm 3$ & $2.0 \pm 0.2$ & $33 \pm 2$ & $36 \pm 2(9)$ & $35 \pm 2$ \\
\hline
\end{tabular}

Notes. In parentheses, the number of objects used for the given average value is indicated. ${ }^{\left({ }^{a}\right)}$ All objects in the sample have measured redshift and photon index (from a simple power law fit). ${ }^{(b)}$ Only objects having variability measured in both the $14-24$ and the $35-100 \mathrm{keV}$ bands have been considered here.

(Fig. 2) and redshifts up to $z=2.5$ (see Fig. 1 in Soldi et al. 2012 for the redshift distribution of the sample). The non-blazar objects belong to the local AGN population with redshifts up to $z=0.1$ and an average value of $\langle z\rangle=0.024$ (Table 1 ).

In Table 2 the values of $S_{V}$ are reported for the 110 AGN selected for the variability amplitude analysis. The blazar population shows a larger average variability $\left(\left\langle S_{V}\right\rangle_{\mathrm{bla}}=33 \pm 2 \%\right)$ compared to the radio quiet objects $\left(\left\langle S_{V}\right\rangle_{\text {Sey }}=19.3 \pm 0.5 \%\right)$. A Kolmogorov-Smirnov test provides a probability $P_{\mathrm{KS}} \geq 98 \%$ that the blazar and Seyfert samples are not drawn from the same parent population. Nevertheless, extreme cases of high variability are present in both the blazar and Seyfert classes. An average variability $S_{V}>90 \%$ is in fact detected in the two gammaray bright blazars QSO B0033+595 and Mrk 421 and in the Seyfert 2 galaxy 2MASX J04440903+2813003.

The radio galaxies present an intermediate behavior between blazars and Seyferts, with $\left\langle S_{V}\right\rangle_{\mathrm{RG}}=24.0 \pm 1.4 \%$. Within the Seyfert class, intermediate and type 2 objects $\left(\left\langle S_{V}\right\rangle_{\text {Sey1.5+2 }}=\right.$ $20.8 \pm 0.6 \%)$ are found to be only slightly more variable than type $1\left(\left\langle S_{V}\right\rangle_{\text {Sey1 }}=16.5 \pm 0.9 \%\right)$, and a KS-test probability $P_{\mathrm{KS}} \geq 94 \%$ indicates that the Seyfert 1 and Seyfert $1.5-$ 2 samples could be drawn from the same parent distribution. Furthermore, no difference is found in the average variability of absorbed and unabsorbed Seyfert galaxies (with $N_{\mathrm{H}}=10^{22} \mathrm{~cm}^{-2}$ the dividing line), with $\left\langle S_{V}\right\rangle_{\mathrm{abs}}=19.7 \pm 0.6 \%$ and $\left\langle S_{V}\right\rangle_{\mathrm{unabs}}=$ $19 \pm 1 \%$. The average properties of the different AGN classes are summarized in Table 1.

\subsection{Black hole mass, hydrogen column density, and Eddington ratio}

To test the dependence of variability on different AGN parameters, we collected the values of black hole masses $M_{\mathrm{BH}}$ and hydrogen column density $N_{\mathrm{H}}$ for our sample from the literature. The masses have been calculated with different methods, and when not available in the literature, the uncertainties on the values were estimated from the typical accuracy provided by the method following Beckmann et al. (2009). Out of the 110 objects, 92 have mass estimates (only an upper limit for MR 2251-178; Table 2), 86 have $N_{\mathrm{H}}$ values, and for 23 only $N_{\mathrm{H}}$ upper limits could be found.

For 63 objects ( 56 Seyferts, 4 radio galaxies, and 3 blazars), we collected the bolometric luminosities from the literature, selecting those estimated from the fitting of the spectral energy distributions (Woo \& Urry 2002; Vasudevan \& Fabian 2007, 2009; Vasudevan et al. 2009, 2010; Table 2). To estimate the bolometric luminosity from the X-ray one, constant corrections (Winter et al. 2009; Beckmann et al. 2009) and corrections that depend on the luminosity (Marconi et al. 2004), on the Eddington ratio (Vasudevan \& Fabian 2007), on the spectral shape (Fabian et al. 2009), or on the intrinsic absorption (Vasudevan et al. 2010) have been proposed, but all fail to reproduce the large scatter of bolometric versus X-ray luminosity. However, to extend the sample for which we are able to compute the Eddington ratio, we also considered two of those bolometric corrections. The first one follows Beckmann et al. (2009), i.e., assumes that the bolometric luminosity $L_{\text {bol }}$ is two times higher than the $1-200 \mathrm{keV}$ luminosity, where $L_{1-200 \mathrm{keV}}=$ $1.8 \times L_{14-195 \mathrm{keV}}$ for a simple power law spectrum with photon index $\Gamma=1.9$. The second one is based on the relation provided by Marconi et al. (2004) to compute $L_{\text {bol }}$ using a luminositydependent bolometric correction. Since these corrections allow having estimates of $L_{\mathrm{bol}}$ for an additional 29 objects (19 Seyfert) and since they trace the bolometric luminosities computed from the SED fitting for our sample sufficiently well, we consider $L_{\text {bol, SED }}$ throughout the paper but compare the results to those obtained with X-ray based $L_{\text {bol }}$ estimates on a larger sample. The Eddington ratio is then computed as $\lambda_{\mathrm{Edd}}=L_{\mathrm{bol}} / L_{\mathrm{Edd}}$, where $L_{\text {Edd }}=1.26 \times 10^{38} M_{\mathrm{BH}} / M_{\odot} \mathrm{erg} \mathrm{s}^{-1}$.

\section{Correlation analysis}

To test for the dependence of the hard X-ray variability on the properties of the selected objects, we correlate the variability estimator $S_{V}$ with the X-ray luminosity $L_{14-195 \mathrm{keV}}$, black hole mass $M_{\mathrm{BH}}$, Eddington ratio $\lambda_{\text {Edd }}$, intrinsic hydrogen column density $N_{\mathrm{H}}$, and spectral shape $\Gamma$. We exclude the radio loud sources from the correlation analysis in order to have a uniform, local Seyfert sample for which it is not necessary to correct variability and luminosity for redshift and beaming effects, and for which the hard X-ray emission is dominated by accretion processes, with no jet contribution. In addition, the results for the Seyfert sample are easily comparable to previous studies that also focus on radio quiet objects.

We do not find any significant correlation of $S_{V}$ with luminosity (Fig. 3) or with absorption (Fig. 4). Variability appears to be marginally anti-correlated with Eddington ratio when using $L_{\text {bol, SED }}$ to compute $\lambda_{\text {Edd }}$, with a Spearman ranking correlation 


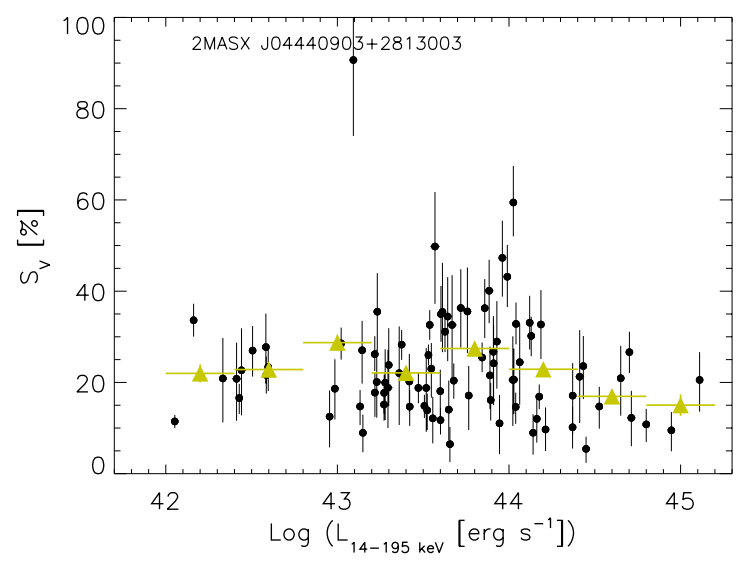

Fig. 3. Variability estimator $S_{V}$ versus luminosity in the $14-195 \mathrm{keV}$ band for the Seyfert galaxies in our sample. Yellow triangles represent the average of the variability estimator in different luminosity bins following Allevato et al. (2013).

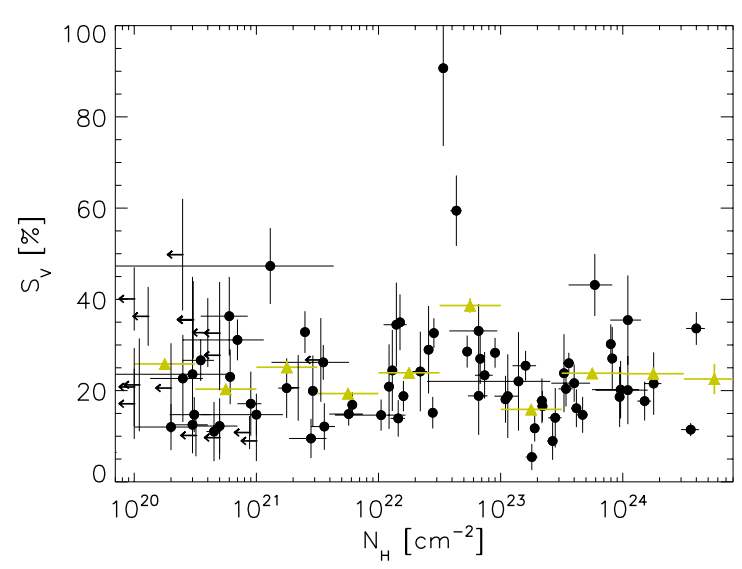

Fig. 4. Variability estimator $S_{V}$ versus intrinsic absorption for the Seyfert galaxy sample. Upper limits on $N_{\mathrm{H}}$ are indicated with arrows. Yellow triangles represent the average of the variability estimator in different absorption bins.

coefficient of $R_{\text {corr }}=-0.3$ corresponding to a probability for chance occurrence of $P_{\text {corr }}=0.03$ (Fig. 5, left panel). However, the correlation disappears when using a larger sample and the $\mathrm{X}$-ray based bolometric corrections to estimate $\lambda_{\text {Edd }}$. When fitting the $S_{V}$ versus Eddington ratio relation with a power law model, we find $S_{V} \propto \lambda_{\text {Edd }}^{-0.044 \pm 0.014}$, which steepens to $S_{V} \propto$ $\lambda_{\text {Edd }}^{-0.09 \pm 0.02}$ when excluding the three sources at super-Eddington rates.

Unlike what is observed in the $2-10 \mathrm{keV}$ band, we do not detect any anti-correlation between the $14-195 \mathrm{keV}$ variability and the black hole mass $\left(R_{\text {corr }}=0.06, P_{\text {corr }}=0.64\right.$; Fig. 6$)$. This agrees with what was found by Shimizu \& Mushotzky (2013) on a smaller sample (Fig. 6). On the other hand, Caballero-Garcia et al. (2012) report a trend toward decreasing variability with increasing black hole mass for the five brightest Seyfert galaxies at hard X-rays, with 20-50 keV BAT light curves rebinned to two days. However, when computing $F_{\text {var }}$ for the two-days binned, 14-195 keV light curves as presented in our work for the same five sources, and applying a Spearman rank correlation test (more robust than the linear/Pearson correlation one; e.g., Press et al. 2007), we do not find any anti-correlation between variability and black hole mass $\left(R_{\mathrm{corr}}=-0.1, P_{\text {corr }}=0.87\right)$.

The lack of an $S_{V}-M_{\mathrm{BH}}$ anti-correlation is most likely because the BAT survey samples time scales that are longer than the power density spectrum (PDS) break, $T_{\mathrm{B}}$. In fact, the X-ray PDS of AGN has a characteristic power law shape with index $\alpha_{\mathrm{PDS}}=-1$ for long time scales and $\alpha_{\mathrm{PDS}}=-2$ for times scales shorter than a source-dependent break time $T_{\mathrm{B}}$ (typically of hours to weeks; Uttley 2005; Arévalo et al. 2008a; Markowitz 2009, 2010; González-Martín \& Vaughan 2012), which is found to scale with black hole mass and bolometric luminosity following the relation described by McHardy et al. (2006). This implies a scaling of the variability amplitude with the same AGN properties when time scales close to or shorter than $T_{\mathrm{B}}$ are considered. Above $15 \mathrm{keV}$ the PDS obtained with BAT data on time scales of weeks to years show for 29 out of 30 objects a single power law with index $\alpha_{\text {PDS }} \sim-0.8$ (SM13), consistent with the 2-10 keV results and providing an upper limit of 26 days for the hard X-ray $T_{\mathrm{B}}$ of these objects. However, if one assumed that $T_{\mathrm{B}}$ is independent of energy and can be estimated using the McHardy et al. (2006) relation, one would find that for 12 Seyferts in our sample $T_{\mathrm{B} \text {, pred }}$ is predicted to be longer than three years. This suggests the possibility that for those AGN, BAT has measured the variability at frequencies above the PDS break and that therefore a scaling of variability with black hole mass should be expected ${ }^{5}$. Nonetheless, no correlation is detected (Fig. 6). Recently, based on the $0.2-10 \mathrm{keV}$ PDS analysis of $104 \mathrm{AGN}$ and on the detection of a break in 15 of them, González-Martín \& Vaughan (2012) have recomputed the $T_{\mathrm{B}}-M_{\mathrm{BH}}-L_{\mathrm{bol}}$ relation finding a weaker dependence on $L_{\mathrm{bol}}$ than in the McHardy et al. (2006) formulation. Therefore, considering the large uncertainties on the mass determination and bolometric luminosity estimate, and on the $T_{\mathrm{B}}-M_{\mathrm{BH}}-L_{\mathrm{bol}}$ relation, it is not possible to derive from the above arguments whether or not this relation is also valid at hard X-rays.

\section{Spectral variability}

\subsection{Variability, photon index, and Eddington ratio}

A significant anti-correlation is found between variability and photon index for Seyfert galaxies, in the sense that AGN with harder spectra are more variable $\left(R_{\text {corr }}=-0.5, P_{\text {corr }}=2 \times 10^{-6}\right.$, Fig. 5 right panel). The photon index used here is the result of a simple power law fit to the BAT spectrum. Even when excluding the objects with $N_{\mathrm{H}}>3 \times 10^{-23} \mathrm{~cm}^{-2}$ for which absorption might start to have a noticeable influence on the hard X-ray spectrum, the anti-correlation is still significant $\left(P_{\text {corr }}=6 \times 10^{-4}\right)$.

A correlation is also identified between photon index and Eddington ratio $\left(R_{\text {corr }}=0.56, P_{\text {corr }}=10^{-5} ;\right.$ Fig. 5 middle panel), similar to what is observed in the $2-10 \mathrm{keV}$ band on year time scales (Sobolewska \& Papadakis 2009). The best fit to the data with a power law model results in $\Gamma \propto \lambda_{\text {Edd }}^{0.022 \pm 0.002}$ $\left(\Gamma \propto \lambda_{\text {Edd }}^{0.039 \pm 0.003}\right.$ when considering only sources with $\left.\lambda_{\text {Edd }}<1\right)$. The correlation is also significant when using the X-ray bolometric corrections to compute $\lambda_{\text {Edd }}$. This relation has been suggested to be based on the geometry of the accretion flow, with stronger accreting objects having accretion disks whose inner radius is closer to the innermost circular stable orbit, so it can cool the hot corona more efficiently, resulting in softer X-ray spectra (Sobolewska \& Papadakis 2009).

The marginal anti-correlation between variability and Eddington ratio (Fig. 5, left panel) could therefore be induced by the combination of the $\Gamma-\lambda_{\text {Edd }}$ and $S_{V}-\Gamma$ ones $\left(S_{V} \propto \Gamma^{-2.5 \pm 0.3}\right.$ for the total Seyfert sample).

5 For the majority of the objects in the SM13 sample (20 out of 23) $T_{\mathrm{B} \text {, pred }}$ is close to or shorter than 30 days, consistent with no $T_{\mathrm{B}}$ detected by SM13 in the BAT time frequency range. 

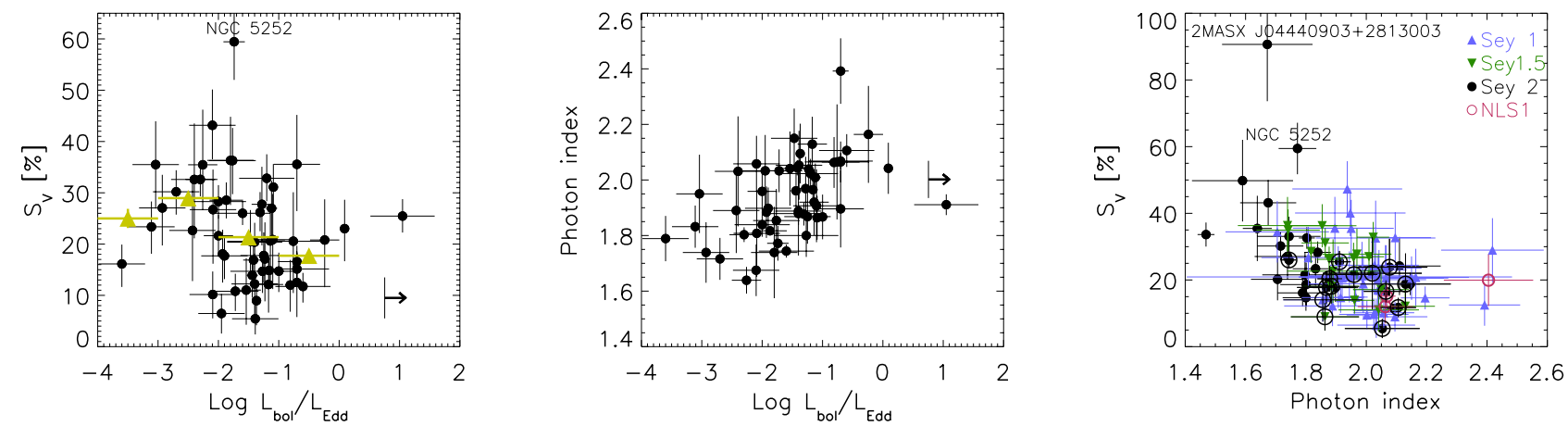

Fig. 5. Left: variability estimator $S_{V}$ versus Eddington ratio for the Seyfert sample. Yellow triangles represent the average of the variability estimator in different Eddington ratio bins. Middle: photon index versus Eddington ratio for the Seyfert sample. Right: variability estimator $S_{V}$ versus photon index for all the Seyfert galaxies in our sample, separated into the different types. Empty black circles indicate those sources with $10^{23}<N_{\mathrm{H}}<4 \times 10^{23} \mathrm{~cm}^{-2}$.

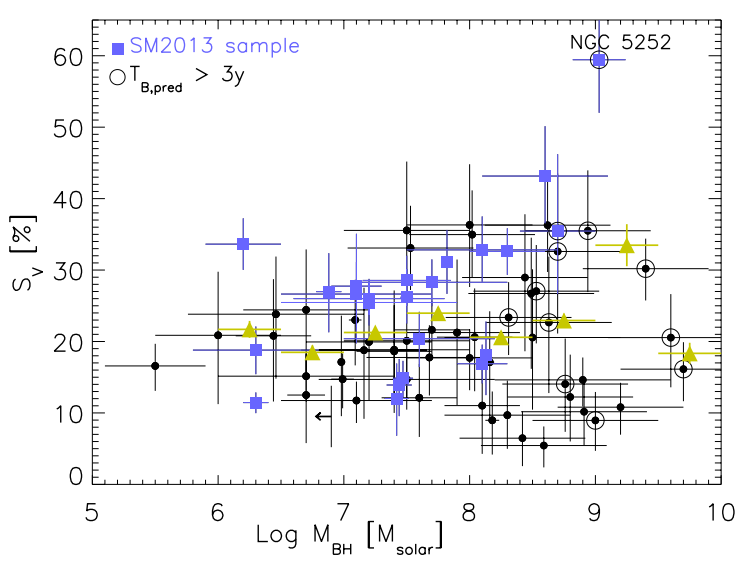

Fig. 6. Variability estimator $S_{V}$ versus black hole mass for the Seyfert galaxies in the sample. Empty circles highlight AGN with predicted break time longer than 3 years, while blue empty squares point out the Seyferts from the SM13 sample. The arrow indicates the $M_{\mathrm{BH}}$ upper limit for MR 2251-178. Yellow triangles represent the average of the variability estimator in different black hole mass bins.

\subsection{Variability in the $14-24$ and $35-100 \mathrm{keV}$ bands}

To compare the variability at different energies, we use the 30-day binned light curves in the 14-24 and 35-100 keV bands (see Table 2). These energy bands were selected to provide comparable significance for a typical hard X-ray AGN spectrum with power law shape and photon index $\Gamma=1.8$.

For a sample of 68 AGN (50 Seyferts, 9 radio galaxies, 9 blazars), we were able to estimate $S_{V}$ for the two selected bands (Fig. 7). In general, the variations are correlated well between the two bands $\left(R_{\text {corr }}=0.6, P_{\text {corr }}=5 \times 10^{-7}\right)$. However, there is an average $10 \%$ shift of the Seyfert population towards higher amplitude of the variations at lower energies, aside from a very few exceptions. A Kolmogorov-Smirnov test indicates a probability $P_{\mathrm{KS}} \geq 98 \%$ that the variations in the two energy bands are different for the 50 Seyferts in this subsample. This is also true when separating the sample into the 13 unabsorbed $\left(N_{\mathrm{H}}<10^{22} \mathrm{~cm}^{-2}\right)$ and 26 absorbed objects: absorbed and unabsorbed sources show the same average variability amplitude, with the lower energy band presenting greater variability $\left(\left\langle S_{V}\right\rangle_{\text {unabs, } 14-24 \mathrm{keV}}=26 \pm 2 \%,\left\langle S_{V}\right\rangle_{\mathrm{abs}, 14-24 \mathrm{keV}}=28 \pm 1 \%\right.$, $\left.\left\langle S_{V}\right\rangle_{\text {unabs,35-100 keV }}=17 \pm 2 \%,\left\langle S_{V}\right\rangle_{\text {abs }, 35-100 \mathrm{keV}}=16 \pm 1 \%\right)$. On the other hand, on average the 18 radio loud AGN do not display a significant dependence of variability on energy.

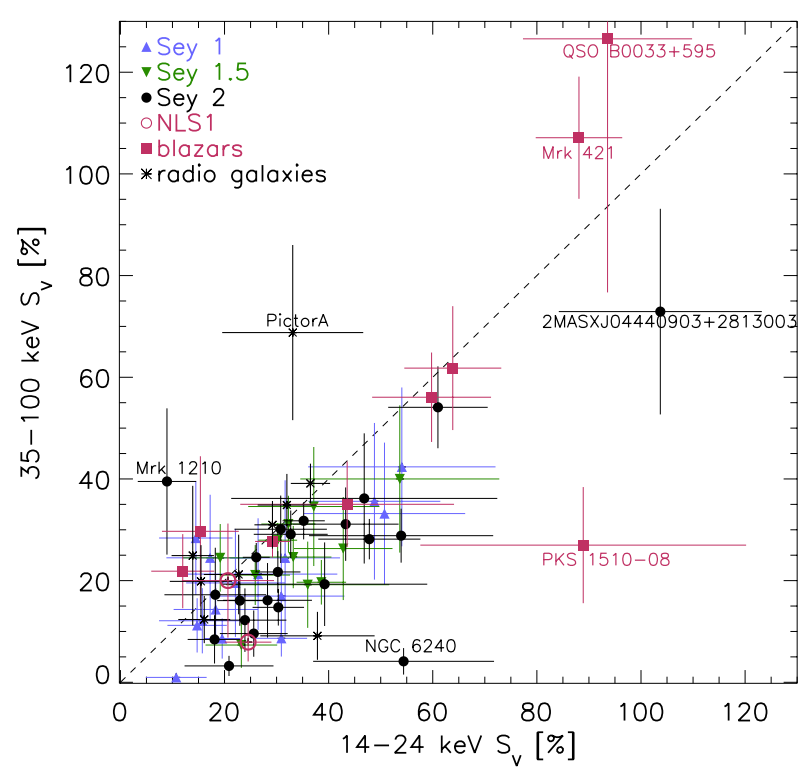

Fig. 7. 35-100 keV versus $14-24 \mathrm{keV}$ variability estimator for the AGN in our sample. The dashed line indicates where objects with the same variability in the two bands would lie. Even though the variations in the two bands are well correlated, the variations in the lower-energy band are larger than those at higher energies for the majority of the radio quiet objects. Some of the most extreme outliers are labeled.

It is important to stress that the 14-24 and 35-100 keV bands have been selected specifically to have similar $\mathrm{S} / \mathrm{N}$ for a typical AGN spectrum, therefore excluding the possibility that the behavior observed could be due to a bias introduced by the different uncertainties in the two bands. In fact, on average the difference between the $\mathrm{S} / \mathrm{N}$ in the low- and high-energy band is $3 \%$ for the total sample $(-1.4 \%$ for the Seyferts and $11 \%$ for the radio loud sources), with about half of the sample having higher $\mathrm{S} / \mathrm{N}$ in the high-energy band.

When testing the possibility that the additional variability in the lower energy band could be ascribed to variations in the intrinsic absorption, we find that large and frequent variations would be needed. In fact, if we consider a simple power law spectrum $(\Gamma=1.9)$ with full-coverage absorption, variations on the order of $\Delta N_{\mathrm{H}} \sim 5-10 \times 10^{23} \mathrm{~cm}^{-2}$ would be needed to produce flux variations of $\Delta F_{14-24 \mathrm{keV}} \sim 8-16 \%$ and $\Delta F_{35-100 \mathrm{keV}}<0.7 \%$. This would imply a $10 \%$ difference between the low- and high-energy flux variations within two observations and correspond to a $\Delta F_{14-195 \mathrm{keV}} \sim 3-6 \%$ contribution to 

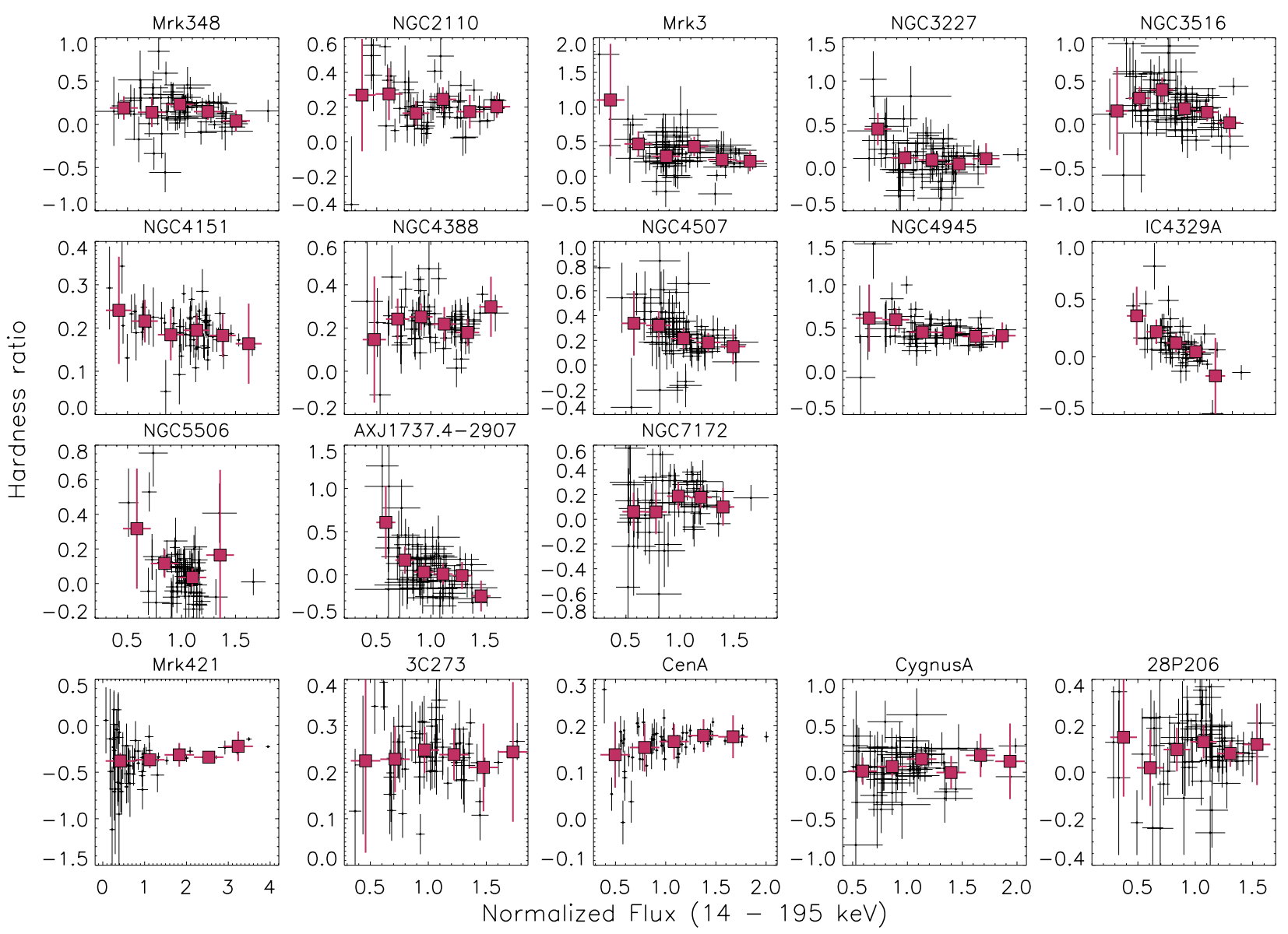

Fig. 8. Hardness ratio versus 14-195 keV flux for the 18 AGN showing spectral variability (13 Seyfert galaxies on the three top rows and 5 radio loud AGN on the bottom row). The black dots are from the 1-month binned light curves, and red squares indicate the average hardness ratio in different flux bins.

the variability in the full band. However, to measure an increase in $S_{V}$ by $10 \%$ given our sampling baseline and rate, unrealistically large $\left(\left\langle\Delta N_{\mathrm{H}}\right\rangle\right.$ at least up to $\left.\sim 10^{25} \mathrm{~cm}^{-2}\right)$ and frequent (on average over $50 \%$ of the light curve points) variations in the absorbing column would be required, and would imply even larger variations in the energy band $<10 \mathrm{keV}$. Even though variable absorbers are indeed common (Risaliti et al. 2002), such extreme variations are very rarely observed (see, e.g., NGC 1365, Risaliti et al. 2007).

\subsection{Hardness ratio versus flux}

To compare the spectral variability within the single sources, for each object we compute the hardness ratio on month time scale, defined as

$H R=\frac{F_{35-100 \mathrm{keV}}-F_{14-24 \mathrm{keV}}}{F_{35-100 \mathrm{keV}}+F_{14-24 \mathrm{keV}}}$.

The dependence of $H R$ on time is then fitted with a constant function, a $\chi^{2}$ test is applied, and the corresponding variability index $V$ is computed (as defined in Sect. 3). Based on this, we select a subsample of 18 objects for which $V>1.3$, as those presenting spectral variability. These are mostly the brightest objects in the sample, with a few exceptions (e.g., MCG-05-23-016, Circinus galaxy). The relatively low fraction of sources showing significant spectral variability agrees with the results presented by Mateos et al. (2007), who find spectral variability to be less common than flux variability on long time scales in the $0.2-12 \mathrm{keV}$ band. In Fig. 8 the hardness ratios as a function of flux (normalized to the mean flux of each object) are presented. For eight Seyferts there is a clear trend toward spectral softening when the source brightens (Mrk 3, NGC 3516, NGC 4151, NGC 4507, NGC 4945, IC 4329A, NGC 5506, AX J1737.4-2907), while no significant correlation is detected for the other radio quiet objects. Our results agree with those reported by Caballero-Garcia et al. (2012) for NGC 4945, NGC 2110, and IC 4329, while different trends are observed for NGC 4388 and NGC 4151. This is possibly due to the different energy bands used to compute HR (20-50 and 50-100 keV in Caballero-Garcia et al. 2012).

There is no correlation between flux and spectral variations for radio loud objects, with the exception of Mrk 421 and Cen A, which exhibit marginal spectral hardening with increasing flux. Cen A has been observed to present a harder-whenbrighter spectrum also during Suzaku observations (Fukazawa et al. 2011). This behavior has been interpreted as due to the increased contribution in the brighter state of a hard tail produced in the jet.

\section{Discussion}

\subsection{Variability of the different AGN classes}

Radio loud objects show larger amplitudes of the variations compared to Seyfert galaxies, as expected if their hard X-ray emission is dominated by the extreme processes taking place in a relativistic jet (e.g., Lichti et al. 2008), while hard X-ray emission from Seyfert galaxies is instead dominated by thermal Comptonization. This is even more evident considering that the 
variability of higher redshift blazars (4 out of 13 objects have $z>0.4$ ) will actually increase when taking the effect of cosmological time dilation into account. Among radio loud objects, the radio galaxies seem to show rather intermediate variability amplitudes between the blazars and the radio quiet class, in agreement with the idea that the jet and the coronal emissions are probably both contributing. This makes it difficult to disentangle the relative importance of these components in the hard X-ray spectra of radio galaxies, as shown, for example, in Centaurus A (Beckmann et al. 2011), 3C 111 (de Jong et al. 2012), and in other radio galaxies (Grandi et al. 2006).

Among Seyfert galaxies, there are indications that type 1.5-2 objects are slightly more variable than type 1 , as already found at hard X-rays in Beckmann et al. (2007a). A similar behavior has been reported by Saxton et al. (2011) when studying long-term X-ray variability properties of a sample of more than 1000 AGN detected by ROSAT and within the XMM-Newton slew survey. Following their study, the Seyfert 2 class has the highest fraction of sources with large variability in the $0.2-2 \mathrm{keV}$ range, but it is not clear if the observed variability is due to changes in the line-of-sight column density, or it is instead intrinsic to the central engine. On the other hand, while in Beckmann et al. (2007a) there was a tentative trend toward more absorbed sources being more variable, here we do not observe any correlation between variability and absorption column density. Therefore, the global properties of variability as a function of AGN class point to general agreement with the basic formulation of AGN unification models, in which the differences are driven in first approximation only by AGN orientation and by the presence or absence of a relativistic jet.

Within our sample, there are three NLS1 galaxies, a class that is known to show peculiar characteristics in the soft X-ray domain, like a steep spectral continuum, and strong and fast variability. Most of these distinctive properties of NLS1s have been interpreted in the context of relatively small black hole masses undergoing rapid evolution, powered by higher accretion rates than their broad line equivalent (Peterson et al. 2000). The average hard X-ray photon index of the NLS1 in our sample is indeed slightly larger than for the rest of the Seyfert class $\left(\langle\Gamma\rangle_{\mathrm{NLS} 1}=2.2 \pm 0.1,\langle\Gamma\rangle_{\mathrm{Sey} 1}=2.01 \pm 0.02\right)$, as also derived from INTEGRAL observations on 14 NLS1s (Ricci et al. 2011b; Panessa et al. 2011). On the other hand, the average variability amplitude is consistent with what is observed for broad line Seyfert $1\left(\left\langle S_{V}\right\rangle_{\mathrm{NLS} 1}=15 \pm 3 \%\right)$. Even though our NLS1 sample is too small to draw any firm conclusion, the discrepancy between the soft and hard X-rays results could be due to the different time scales probed in these studies: while NLS1s do show strong variability on short time scales, on long time scales above the PDS time break, their timing characteristics do not differ significantly from those of the general type-1 Seyfert population. This agrees with Saxton et al. (2011), who find substantially the same long-term variability in narrow and broad line Seyfert 1 within their $0.2-2 \mathrm{keV}$ survey study.

\subsection{Long-term variability, luminosity and black hole mass}

Due to the rather long time scales probed by BAT, the anticorrelations of variability with luminosity (Barr \& Mushotzky 1986; Green et al. 1993; Papadakis 2004) and with black hole mass (Papadakis \& McHardy 1995; Zhou et al. 2010; Kelly et al. 2011, 2013; Ponti et al. 2012) often observed below $10 \mathrm{keV}$ on short time scales are not detected in our hard X-ray study (Figs. 3 and 6). This agrees with results obtained in the soft $\mathrm{X}$-ray band when year time scales are investigated. For example,
Saxton et al. (2011) do not find any relation between long-term variability and luminosity when studying a $0.2-2 \mathrm{keV}$ AGN sample and argue that this is expected since the sampled time scales are substantially longer than the typical PDS break times of AGN. Moreover, Markowitz \& Edelson (2004) analyzed a sample of 27 AGN with ASCA and RXTE data in the 2-12 keV energy range. Even though they do detect anti-correlations between variability and luminosity and variability and black hole mass on day time scales, the significance of these relations decreases when studying longer time scales (a month to years), and the slope of the linear fit to the data approaches zero, indicating that there is no anti-correlation observed on long time scales. Zhang (2011) used RXTE/ASM data, monitoring 27 AGN for about 14 years in the $1.5-12 \mathrm{keV}$ band. The excess variance computed on the 300-day binned light curves does not correlate with the black hole mass. The author argues that this points to AGN being in a high/soft state rather than in a low/hard state, based on an analogy with Galactic black holes (GBH) when solely their timing behavior is considered. In fact, PDS of GBHs in low/hard state are observed to present a second break at even lower time frequencies, and therefore if a similar behavior has to be expected from AGN, a mass dependence of the excess variance should also be observed on long time scales, which is instead not detected.

Finally, considering that the BAT PDS on long time scales appear to have a slope consistent with $\alpha_{\mathrm{PDS}} \sim 1$ (SM13), $S_{V}$ computed in this time frequency range is proportional to the PDS normalization. Therefore, the lack of correlation between $S_{V}$ and $M_{\mathrm{BH}}$ and $L_{\mathrm{X}}$ indicates that the PDS normalization does not seem to scale with black hole mass or luminosity. Moreover, the average variability for Seyfert galaxies is $\left\langle S_{V}\right\rangle_{\text {Sey }}=19 \%$ with $68 \%$ of the objects having $S_{V}$ values in the range $\sim 10-35 \%$. The total variability range is smaller by a factor of $\sim 6$ with respect to the range measured on shorter time scales by Ponti et al. (2012) in the $2-10 \mathrm{keV}$ energy band. This suggests that the BAT $S_{V}$, hence the normalization of the PDS on long time scales, could be very similar in all local Seyfert galaxies.

\subsection{Spectral variability}

A significant correlation is detected between variability in the 14-24 and 35-100 keV energy ranges, with Seyfert galaxies being on average more variable in the lower energy band (Fig. 7). The detected correlation suggests that the same process is likely to be at the origin of the variability in the two bands. Moreover, the presence of such large average variations, even in the 35-100 keV range, is incompatible with hard X-ray variability being entirely due to variations in the hydrogen column density along the line of sight. In fact, as an indication, a variation of $\Delta N_{\mathrm{H}} \sim 5 \times 10^{24} \mathrm{~cm}^{-2}$ would imply only a $3 \%$ variation in the $35-100 \mathrm{keV}$ flux, yet a limited fraction of the additional variability observed at lower (14-24 keV) energies could be due to absorption variations.

Several studies have detected decreasing variability with increasing X-ray energies in the $0.2-12 \mathrm{keV}$ band, either through excess variance or PDS amplitude (e.g., Nandra 2001; McHardy et al. 2004; Markowitz \& Edelson 2004; Ponti 2007; Ponti et al. 2012), and both on short ( $\lesssim 1$ day) and long time scales ( $\gtrsim 30$ days). The same finding extends to hard X-ray energies, with the 14-24 keV band showing on average larger variations than the 35-100 keV one in Seyfert galaxies. A similar result has been obtained by Chitnis et al. (2009) when comparing the 1.5-12 keV RXTE/ASM variability to that measured in the 14-195 keV band by BAT. Shimizu \& Mushotzky (2013) did not 
detect an energy dependence of the PDS parameters for the three Seyferts for which this investigation was possible, but the corresponding excess variance (computed using the PDS parameters) decreases through the 14-24, 24-50, and 50-150 keV bands they studied. If for observations below $10 \mathrm{keV}$ changes of the amount of the absorbing material or of its ionization could play a role (Risaliti et al. 2002; Ponti et al. 2012), in the BAT band such mechanisms are unlikely to be able to fully explain the observed properties.

The variations could instead originate directly at the X-ray source. For example, if a power law spectrum pivots at energies $\gtrsim 50 \mathrm{keV}$, the $14-24 \mathrm{keV}$ flux would be expected to vary more strongly than the $35-100 \mathrm{keV}$ one (Gierliński \& Zdziarski 2005). If we assume a standard Comptonization model based on a two-phase accretion disk (Haardt \& Maraschi 1993), the hard $\mathrm{X}$-ray variability could be driven by changes in the seed photon flux (Nandra et al. 2000; Arévalo et al. 2005) or by physical variations in the corona (temperature and optical depth; Nicastro et al. 2000; Lubiński et al. 2010). Petrucci et al. (2000) point out that in order to have a pivoting point at high energies an increase in the cooling is required rather than a decrease in the heating, which would instead determine a low-energy pivoting point.

In addition, the superposition of two components, one constant (e.g., the reflected continuum) and the other one variable (for example, the Comptonized continuum) could contribute further to the observed spectral variations (Shih et al. 2002; Markowitz \& Edelson 2004; Ponti et al. 2006; Papadakis et al. 2009; Sobolewska \& Papadakis 2009). In the 14-24 keV and $35-100 \mathrm{keV}$ bands, the contribution of reflection to the observed flux is about $30-40 \%$ for a reflection fraction $R=1$ and typical AGN spectra with $\Gamma=1.6-2.1, N_{\mathrm{H}}=1-100 \times 10^{22} \mathrm{~cm}^{-2}$, and a high-energy cut-off at $E_{\mathrm{C}}=100-300 \mathrm{keV}$. For such spectra, a larger contribution from reflection is measured in the total 35$100 \mathrm{keV}$ flux compared to the $14-24 \mathrm{keV}$ one, but only by a few percentage points. Therefore, reflection alone cannot fully explain the difference observed in the two hard X-ray bands, even though a contribution from it is expected.

The scenario of a pivoting spectrum superposed on a constant reflection is in general also consistent with a steepening of the hard X-ray spectrum when the source brightens. This is indeed observed for the majority of the Seyferts in our sample that show some spectral variability (Fig. 8) and follows an established trend observed in the X-ray emission of numerous local radio quiet AGN (Markowitz \& Edelson 2004; Sobolewska \& Papadakis 2009; Caballero-Garcia et al. 2012) and also in higher redshift objects (Paolillo et al. 2004). Within the Comptonization frame, the softer-when-brighter behavior can be understood if an increase in the seed photon power illuminating the corona determines a more efficient cooling of the hot electrons, with a resulting drop in the coronal temperature, which causes the X-ray spectrum to steepen.

The correlation between Eddington ratio and photon index is another known property of AGN samples below $10 \mathrm{keV}$ (Sobolewska \& Papadakis 2009), and it is confirmed by our study at hard X-rays (Fig. 5, middle panel), in agreement with Middleton et al. (2008), who suggest this relation to explain possible differences in hard X-ray spectral slopes for different AGN classes.

A different behavior arises when comparing the hard X-ray to the soft X-ray variability. We collected from the literature the PDS parameters for 13 AGN in our sample, i.e., those objects with measured soft X-ray PDS and with detected frequency break (González-Martín \& Vaughan 2012, SM13, and references therein). We compare the normalized excess variance $\sigma_{\mathrm{rms}}^{2}$ in

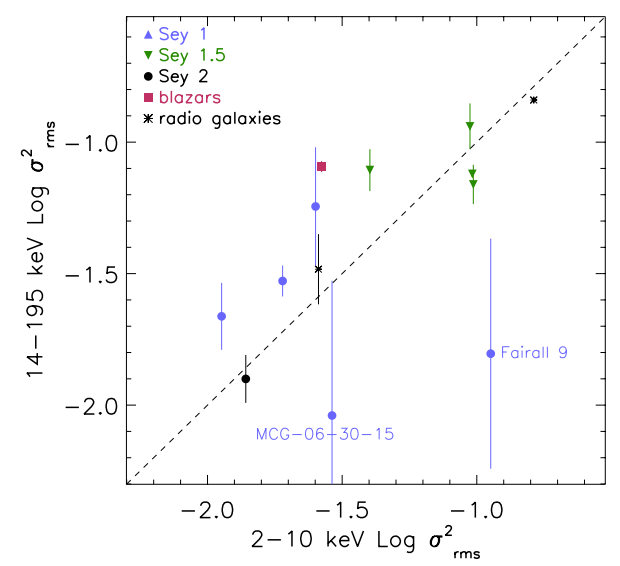

Fig. 9. $14-195 \mathrm{keV}$ versus $2-10 \mathrm{keV}$ normalized excess variance $\sigma_{\mathrm{rms}}^{2}$ for 10 Seyfert galaxies and 3 radio loud AGN (3C 273, 3C 390.3, Cen A) in our sample for which $2-10 \mathrm{keV}$ PDS with a measured frequency break have been reported in the literature. The dashed line indicates where objects with the same variability at soft and hard X-rays would lie.

the $14-195 \mathrm{keV}$ band to the $2-10 \mathrm{keV}$ one, computed by extrapolating the soft X-ray PDS to low frequencies in order to cover the same time scales as the BAT data set, i.e., between $v_{\min }=6 \times 10^{-9} \mathrm{~Hz}$ and $v_{\max }=4 \times 10^{-7} \mathrm{~Hz}$. The variability amplitude at hard X-rays is found to be equal to or larger than the one in the soft X-rays (Fig. 9). This is consistent with what has been found by Caballero-Garcia et al. (2012) and Shimizu \& Mushotzky (2013), yet it is surprising, since it seems to be an opposite trend to those observed within the soft and hard $\mathrm{X}$-rays bands alone. One reason could be that, when extrapolating the 2-10 keV PDS to year time scales, the uncertainties on the measurement of the PDS slope introduce a large scatter in the estimated excess variance. Indeed, for 6 out of 13 objects, the low-frequency PDS slope has been fixed to $\alpha=-1$ (from González-Martín \& Vaughan 2012). Slightly steeper slopes by $\Delta \alpha=0.1-0.2$ would imply a comparable or larger variability at soft rather than at hard X-rays.

If instead the hard X-rays are indeed more variable than the soft X-rays on long time scales, this might indicate an even more complex dependence of variability on energy. The difference between soft and hard X-ray variability could be, for example, related to the size of the emitting region. If the highenergy plasma is located inside the accretion disk or above its inner part (Zdziarski et al. 1999; Lubiński et al. 2010), while the low-energy emitting region is associated to some outflow from the disk or a second plasma with lower temperature (Petrucci et al. 2013), then the high-energy emission could be more variable than the lower-energy emission. However, the change in relative dominance of the two components would have to happen exactly at energies of 10-15 keV. In addition, it would be necessary to explain why this trend is inverted within the soft and hard X-ray bands, with the higher energy emission in general less variable than the lower energy emission.

\subsubsection{Variability and photon index anti-correlation}

Another interesting difference compared to the lower energies and lower time frequencies is the trend of harder sources to be more variable at hard X-rays (Fig. 5, right panel). In fact, several papers report the opposite trend for softer sources to be more variable on time scales close to or below a day, even though with different degrees of confidence. O'Neill et al. (2005) find a marginal correlation between photon index and excess variance, 


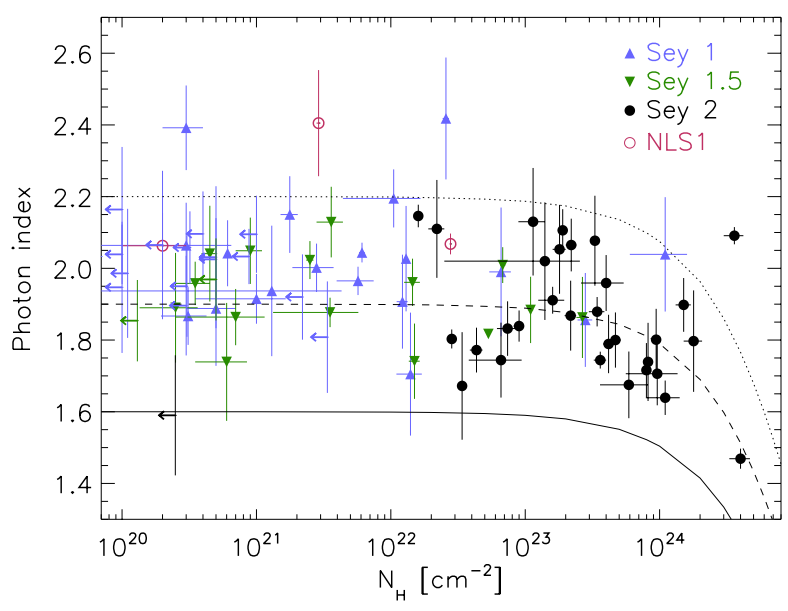

Fig. 10. Measured photon index versus intrinsic absorption for the Seyfert galaxy sample. The lines correspond to the measured photon index obtained from a simple power law fit of simulated power law spectra with intrinsic photon index $\Gamma=1.6,1.9$, and 2.2 (from bottom to top) modified by the corresponding $N_{\mathrm{H}}$. Upper limits on $N_{\mathrm{H}}$ are indicated with arrows.

while Green et al. (1993) and Grupe et al. (2001) find a more significant correlation in the $1-8 \mathrm{keV}$ (EXOSAT) and $0.2-2 \mathrm{keV}$ band (ROSAT), respectively. One has to keep in mind, though, that a contribution from absorption variations could be important in the ROSAT data. The largest soft X-ray sample for this kind of study has been presented by Ponti et al. (2012). They confirm the correlation between excess variance and photon index, which they ascribe to the fact that accretion rate correlates with both variability and photon index in their sample. In contrast, at hard X-rays and low time frequency, it seems more likely that the correlation between accretion rate and photon index and the anti-correlation between photon index and variability are the origin of the marginal anti-correlation between accretion rate and variability (Fig. 5).

The photon index versus variability anti-correlation is instead commonly observed in Galactic black holes (GBH), which show larger variability during the hard state, i.e., when the corona rather than the accretion disk emission is believed to dominate the X-ray spectrum (e.g., Muñoz-Darias et al. 2011). Moreover, a decreasing variability with the softening of the spectrum is detected in the so-called hard-intermediate state, when the less variable accretion disk component starts contributing to the X-ray spectrum of GBHs (Belloni et al. 2011). This might indicate that the larger variability in the harder BAT sources could be associated to their stronger Comptonization.

The anti-correlation between variability and photon index in our sample could, at least partly, be induced by the effect of different reflection fractions. On one hand, if one assumes that the bulk of reflection is mostly constant, objects with larger reflection would be expected to be less variable. Constant or slowly variable reflection components observed in a number of objects have been explained as either due to a distant reflector, with light travel time damping out variability (e.g., Miller et al. 2008; Bianchi et al. 2009), or as a consequence of strong gravitational light bending in the close vicinity of the black hole (e.g., Miniutti \& Fabian 2004; Ponti et al. 2006; Miniutti et al. 2007). On the other hand, there are indications that objects with larger reflection have softer X-ray spectra. In fact, Zdziarski et al. (1999) reported a strong correlation indicating that intrinsically softer sources have larger reflection fractions, possibly due to the reflector also being the source of seed photons for the Comptonization emission. In addition to this effect, when fitting with a simple power law model spectra with different reflection strengths and the same intrinsic continuum, the measured photon index in the 14-195 keV band results steeper for larger reflection, with $\Delta \Gamma \lessgtr 7 \%$ (depending on the intrinsic continuum shape and on the position of the high-energy spectral turnover) for a reflection fraction $R$ changing from 0 to 2 . Reflection fractions $R>1$ might be the result of peculiar geometries (causing, e.g., high covering factors; Malzac 2001), of light bending effects in the vicinity of the black hole (Miniutti \& Fabian 2004), or of a variable continuum combined with a delayed answer of the reflected component, due to the distance of the reflector (Malzac $\&$ Petrucci 2002).

When the accretion disk is considered as the main reflector, a larger reflection component is expected from face-on compared to edge-on AGN. Indeed, when dividing our sample into the different AGN classes, Seyfert 1 appear at the higher end of the photon index distribution, consistent with larger reflection being associated to softer spectra. Furthermore, Ricci et al. (2011b) and Vasudevan et al. (2013) have recently shown that objects with absorption in the range $10^{23}<N_{\mathrm{H}}<10^{24} \mathrm{~cm}^{-2}$ present a stronger reflection component than those at lower $N_{\mathrm{H}}$. When looking at the distribution of photon indexes as a function of $N_{\mathrm{H}}$, sources in the range $10^{23}<N_{\mathrm{H}}<4 \times 10^{23} \mathrm{~cm}^{-2}$ have a softer spectrum than the rest of the Seyfert 2 population and comparable to the average Seyfert 1 one (Fig. 10), possibly pointing out a stronger reflection component. We note that the photon index drop at $N_{\mathrm{H}}>4 \times 10^{23} \mathrm{~cm}^{-2}$ cannot be simply explained by our fitting not taking absorption into account. In fact, when simulating absorbed spectra and fitting them with a simple power law, the measured photon index decreases much less dramatically as a function of $N_{\mathrm{H}}$ than is observed in the data (see lines in Fig. 10).

However, caution should be used since for highly absorbed sources simple absorption models fail to correctly reproduce the spectra in the presence of strong reprocessed emission (Lubinski et al., in prep.; Yaqoob 2012; Braito et al. 2013), and since stacked spectra might be influenced by the effects of combining objects with different spectral shapes contributing with varying weight to the different energy bands. In addition, the validity of the $\Gamma-R$ correlation reported by Zdziarski et al. (1999) is still being debated. Some later studies have confirmed this relation (Mattson et al. 2007; Dadina 2008; Winter et al. 2012), whereas others did not (Molina et al. 2009; de Rosa et al. 2012), pointing out the difficulty in disentangling a real physical relation from the degeneracies among photon index, reflection, and high-energy cut-off in the commonly used X-ray fitting models (Mattson et al. 2007; Winter et al. 2012).

An alternative explanation could come from a similar trend observed in NGC 7469 (Nandra \& Papadakis 2001), which presented a higher excess variance when the spectrum of the source was harder. Nandra \& Papadakis (2001) suggest that this and other timing properties of this AGN could be explained if the inner regions of the corona were hotter than the outer ones. The hard X-ray emission would then be produced in the innermost parts of the corona and therefore be more variable and have shorter time scales than the soft X-ray one, produced in the larger and cooler outer zone. However, these results are based on a 30-day observation at $<15 \mathrm{keV}$, so the time scales and energies are different than those of our work. Similarly, in the model first proposed by Lyubarskii (1997) and then reconsidered by others (Churazov et al. 2001; Arévalo \& Uttley 2006), different variability patterns at different energies and on different time scales are explained with accretion disk fluctuations propagating inward and illuminating a corona with emissivity gradient, which would therefore modulate the X-ray source. 


\section{Conclusions}

We have characterized the $14-195 \mathrm{keV}$ variability of the largest sample of hard X-ray-selected AGN provided by Swift/BAT to date. The amplitude of the variations was quantified and their dependence on energy and on basic AGN properties, such as black hole mass, luminosity, Eddington ratio, and intrinsic absorption, were investigated. The average characteristics of hard $\mathrm{X}$-ray variability generally agree with the unified models, indicating larger variability in jetted sources and no clear dependence of the variations on AGN orientation. The amplitude of the hard X-ray variations and its spectral properties indicate that variability cannot be driven by absorption variations. On the other hand for radio quiet AGN, it is rather consistent with changes of a power law continuum over a constant component, with the power law pivoting around $\gtrsim 50 \mathrm{keV}$. Such variations in the continuum emission would be achieved for example with a change in the corona temperature, determining a shift of the high-energy cut-off at hard X-rays, either due to intrinsic coronal variations or to a change in the seed photon flux.

In general, variability at hard X-rays has very similar properties to that in the softer X-ray band, when similar time scales are compared, compatible with variability being driven by the same mechanism across the full X-ray band. However, some discrepancies, like AGN with harder spectra being more variable and the apparent lower variability in the $2-10 \mathrm{keV}$ band, could be ascribed to additional components in either band (e.g., reflection, absorption), and in any case they could provide interesting insight into the intrinsic differences of the emission in the soft and hard X-ray bands, such as for the emitting regions and their sizes.

The lack of dependence of the long-term variability on black hole mass and luminosity, and the relatively narrow range of variability measured in Seyfert galaxies, suggest that the normalization of the X-ray power spectrum is independent of black hole mass and luminosity and might have a similar value for all local Seyfert galaxies.

Thanks to the Swift/BAT ongoing observations, it will be possible to further extend the studied time scales and increase the available AGN sample, obtaining the best hard X-ray AGN variability sample for some time to come. At the same time, pointed observations with Suzaku, INTEGRAL, and NuSTAR will continue to investigate the spectral variability in bright and well studied AGN, as already successfully done in a number of sources (Reeves et al. 2007; Itoh et al. 2008; Terashima et al. 2009; Fukazawa et al. 2011; Beckmann et al. 2007b; Soldi et al. 2010, 2011b; Lubiński et al. 2010).

Acknowledgements. The authors thank Jerome Rodriguez for useful discussions of Galactic black holes, and the anonymous referee for the valuable suggestions that helped to improve this paper. S.S. acknowledges the Centre National d'Études Spatiales (CNES) for financial support. G.P. acknowledges support via an EU Marie Curie Intra-European fellowship under contract No. FPPEOPLE-2012-IEF-331095. This work has been partly supported by the LabEx UnivEarthS ${ }^{6}$ project "Impact of black holes on their environment", and by the Polish NCN grants N N203 581240 and 2012/04/M/ST9/00780. This article commemorates our colleague Jack Tueller who passed away during the study.

\section{References}

Allevato, V., Paolillo, M., Papadakis, I., \& Pinto, C. 2013, ApJ, 771, 9 Almaini, O., Lawrence, A., Shanks, T., et al. 2000, MNRAS, 315, 325 Arévalo, P., \& Uttley, P. 2006, MNRAS, 367, 801

Arévalo, P., Papadakis, I., Kuhlbrodt, B., \& Brinkmann, W. 2005, A\&A, 430, 435

6 http://wWw . univearths. fr/en
Arévalo, P., McHardy, I. M., \& Summons, D. P. 2008a, MNRAS, 388, 211

Arévalo, P., Uttley, P., Kaspi, S., et al. 2008b, MNRAS, 389, 1479

Barr, P., \& Mushotzky, R. F. 1986, Nature, 320, 421

Barthelmy, S. D., Barbier, L. M., Cummings, J. R., et al. 2005, Space Sci. Rev., 120,143

Baumgartner, W. H., Tueller, J., Markwardt, C. B., et al. 2013, ApJS, 207, 19

Beckmann, V., \& Shrader, C. R. 2012, Active Galactic Nuclei (Wiley-VCH)

Beckmann, V., Barthelmy, S. D., Courvoisier, T. J.-L., et al. 2007a, A\&A, 475

Beckmann, V., Gehrels, N., \& Tueller, J. 2007b, ApJ, 666, 122

Beckmann, V., Courvoisier, T. J.-L., Gehrels, N., et al. 2008, A\&A, 492, 93

Beckmann, V., Soldi, S., Ricci, C., et al. 2009, A\&A, 505, 417

Beckmann, V., Jean, P., Lubiński, P., Soldi, S., \& Terrier, R. 2011, A\&A, 531, A70

Bell, M. E., Tzioumis, T., Uttley, P., et al. 2011, MNRAS, 411, 402

Belloni, T. M., Motta, S. E., \& Muñoz-Darias, T. 2011, Bull. Astron. Soc. Ind. 39, 409

Bian, W., \& Gu, Q. 2007, ApJ, 657, 159

Bianchi, S., Piconcelli, E., Chiaberge, M., et al. 2009, ApJ, 695, 781

Braito, V., Ballo, L., Reeves, J. N., et al. 2013, MNRAS, 428, 2516

Breedt, E., McHardy, I. M., Arévalo, P., et al. 2010, MNRAS, 403, 605

Caballero-Garcia, M. D., Papadakis, I. E., Nicastro, F., \& Ajello, M. 2012, A\&A, 537, A87

Chatterjee, R., Marscher, A. P., Jorstad, S. G., et al. 2011, ApJ, 734, 43

Chitnis, V. R., Pendharkar, J. K., Bose, D., et al. 2009, ApJ, 698, 1207

Churazov, E., Gilfanov, M., \& Revnivtsev, M. 2001, MNRAS, 321, 759

Cusumano, G., La Parola, V., Segreto, A., et al. 2010, A\&A, 524, A64

Czerny, B., Nikołajuk, M., Piasecki, M., \& Kuraszkiewicz, J. 2001, MNRAS, 325,865

Dadina, M. 2008, A\&A, 485, 417

de Jong, S., Beckmann, V., \& Mattana, F. 2012, A\&A, 545, A90

De Marco, B., Ponti, G., Cappi, M., et al. 2013, MNRAS, 431, 2441

de Rosa, A., Piro, L., Perola, G. C., et al. 2007, A\&A, 463, 903

de Rosa, A., Panessa, F., Bassani, L., et al. 2012, MNRAS, 420, 2087

Denney, K. D., Peterson, B. M., Pogge, R. W., et al. 2010, ApJ, 721, 715

Fabian, A. C., Vasudevan, R. V., Mushotzky, R. F., Winter, L. M., \& Reynolds,

C. S. 2009, MNRAS, 394, L89

Fukazawa, Y., Hiragi, K., Yamazaki, S., et al. 2011, ApJ, 743, 124

Gehrels, N., Chincarini, G., Giommi, P., et al. 2004, ApJ, 611, 1005

Gierliński, M., \& Zdziarski, A. A. 2005, MNRAS, 363, 1349

González-Martín, O., \& Vaughan, S. 2012, A\&A, 544, A80

Grandi, P., Malaguti, G., \& Fiocchi, M. 2006, ApJ, 642, 113

Green, A. R., McHardy, I. M., \& Lehto, H. J. 1993, MNRAS, 265, 664

Greenhill, L. J., Booth, R. S., Ellingsen, S. P., et al. 2003, ApJ, 590, 162

Grier, C. J., Peterson, B. M., Pogge, R. W., et al. 2012, ApJ, 755, 60

Grupe, D., Thomas, H.-C., \& Beuermann, K. 2001, A\&A, 367, 470

Haardt, F., \& Maraschi, L. 1993, ApJ, 413, 507

Harmon, B. A., Wilson, C. A., Fishman, G. J., et al. 2004, ApJS, 154, 585

Itoh, T., Done, C., Makishima, K., et al. 2008, PASJ, 60, 251

Kaspi, S., Brandt, W. N., Maoz, D., et al. 2007, ApJ, 659, 997

Kelly, B. C., Sobolewska, M., \& Siemiginowska, A. 2011, ApJ, 730, 52

Kelly, B. C., Treu, T., Malkan, M., Pancoast, A., \& Woo, J.-H. 2013, ApJ, 779, 187

Körding, E. G., Migliari, S., Fender, R., et al. 2007, MNRAS, 380, 301

Krimm, H. A., Holland, S. T., Corbet, R. H. D., et al. 2013, ApJS, 209, 14

Lanzuisi, G., Ponti, G., Salvato, M., et al. 2013, ApJ, submitted

Lichti, G. G., Bottacini, E., Ajello, M., et al. 2008, A\&A, 486, 721

Lu, Y., \& Yu, Q. 2001, MNRAS, 324, 653

Lubiński, P., Zdziarski, A. A., Walter, R., et al. 2010, MNRAS, 408, 1851

Lyubarskii, Y. E. 1997, MNRAS, 292, 679

Malizia, A., Bassani, L., Bird, A. J., et al. 2008, MNRAS, 389, 1360

Malzac, J. 2001, MNRAS, 325, 1625

Malzac, J., \& Petrucci, P.-O. 2002, MNRAS, 336, 1209

Marconi, A., Risaliti, G., Gilli, R., et al. 2004, MNRAS, 351, 169

Markowitz, A. 2009, ApJ, 698, 1740

Markowitz, A. 2010, ApJ, 724, 26

Markowitz, A., \& Edelson, R. 2004, ApJ, 617, 939

Mateos, S., Barcons, X., Carrera, F. J., et al. 2007, A\&A, 473, 105

Mattson, B. J., Weaver, K. A., \& Reynolds, C. S. 2007, ApJ, 664, 101

McHardy, I. 2010, in Lect. Notes Phys. 794, ed. T. Belloni (Berlin Springer Verlag), 203

McHardy, I. M., Papadakis, I. E., Uttley, P., Page, M. J., \& Mason, K. O. 2004, MNRAS, 348, 783

McHardy, I. M., Koerding, E., Knigge, C., Uttley, P., \& Fender, R. P. 2006, Nature, 444, 730

McHardy, I., Lawson, A., Newsam, A., et al. 2007, MNRAS, 375, 1521

Mehdipour, M., Branduardi-Raymont, G., Kaastra, J. S., et al. 2011, A\&A, 534, A39 
Middleton, M., Done, C., \& Schurch, N. 2008, MNRAS, 383, 1501

Miller, L., Turner, T. J., \& Reeves, J. N. 2008, A\&A, 483, 437

Miniutti, G., \& Fabian, A. C. 2004, MNRAS, 349, 1435

Miniutti, G., Fabian, A. C., Anabuki, N., et al. 2007, PASJ, 59, 315

Molina, M., Bassani, L., Malizia, A., et al. 2009, MNRAS, 399, 1293

Muñoz-Darias, T., Motta, S., \& Belloni, T. M. 2011, MNRAS, 410, 679

Nandra, K. 2001, in Probing the Physics of Active Galactic Nuclei, eds. B. M. Peterson, R. W. Pogge, \& R. S. Polidan, ASP Conf. Ser., 224, 167

Nandra, K., \& Papadakis, I. E. 2001, ApJ, 554, 710

Nandra, K., Le, T., George, I. M., et al. 2000, ApJ, 544, 734

Nicastro, F., Piro, L., De Rosa, A., et al. 2000, ApJ, 536, 718

Nikolajuk, M., Papadakis, I. E., \& Czerny, B. 2004, MNRAS, 350, L26

O’Neill, P. M., Nandra, K., Papadakis, I. E., \& Turner, T. J. 2005, MNRAS, 358, 1405

Padovani, P., \& Rafanelli, P. 1988, A\&A, 205, 53

Panessa, F., de Rosa, A., Bassani, L., et al. 2011, MNRAS, 417, 2426

Paolillo, M., Schreier, E. J., Giacconi, R., Koekemoer, A. M., \& Grogin, N. A. 2004, ApJ, 611, 93

Papadakis, I. E. 2004, MNRAS, 348, 207

Papadakis, I. E., \& McHardy, I. M. 1995, MNRAS, 273, 923

Papadakis, I. E., Sobolewska, M., Arevalo, P., et al. 2009, A\&A, 494, 905

Peterson, B. M., McHardy, I. M., Wilkes, B. J., et al. 2000, ApJ, 542, 161

Petrucci, P. O., Haardt, F., Maraschi, L., et al. 2000, ApJ, 540, 131

Petrucci, P.-O., Paltani, S., Malzac, J., et al. 2013, A\&A, 549, A73

Pian, E., Foschini, L., Beckmann, V., et al. 2006, A\&A, 449, L21

Pian, E., Ubertini, P., Bazzano, A., et al. 2011, A\&A, 526, A125

Ponti, G. 2007, Ph.D. Thesis, Università di Bologna

Ponti, G., Cappi, M., Dadina, M., \& Malaguti, G. 2004, A\&A, 417, 451

Ponti, G., Miniutti, G., Cappi, M., et al. 2006, MNRAS, 368, 903

Ponti, G., Papadakis, I., Bianchi, S., et al. 2012, A\&A, 542, A83

Press, W. H., Teukolsky, S. A., Vetterling, W. T., \& Flannery, B. P. 2007, Numerical recipes: The art of scientific computing

Reeves, J. N., Awaki, H., Dewangan, G. C., et al. 2007, PASJ, 59, 301

Reis, R. C., Fabian, A. C., Reynolds, C. S., et al. 2012, ApJ, 745, 93

Ricci, C., Paltani, S., Soldi, S., \& Courvoisier, T. J. L. 2011a, in Fast X-ray Timing and Spectroscopy at Extreme Count Rates (HTRS 2011) [arXiv: 1106.3997]

Ricci, C., Walter, R., Courvoisier, T. J.-L., \& Paltani, S. 2011b, A\&A, 532, A102

Risaliti, G., Elvis, M., \& Nicastro, F. 2002, ApJ, 571, 234

Risaliti, G., Elvis, M., Fabbiano, G., et al. 2007, ApJ, 659, L111

Saxton, R., Read, A., Esquej, P., Miniutti, G., \& Alvarez, E. 2011, in Narrow-Line Seyfert 1 Galaxies and their Place in the Universe PoS(NLS1)008
Shemmer, O., Uttley, P., Netzer, H., \& McHardy, I. M. 2003, MNRAS, 343, 1341

Shih, D. C., Iwasawa, K., \& Fabian, A. C. 2002, MNRAS, 333, 687

Shimizu, T. T., \& Mushotzky, R. F. 2013, ApJ, 770, 60, SM13

Simpson, G., \& Mayer-Hasselwander, H. 1986, A\&A, 162, 340

Sobolewska, M. A., \& Papadakis, I. E. 2009, MNRAS, 399, 1597

Soldi, S., Türler, M., Paltani, S., et al. 2008, A\&A, 486, 411

Soldi, S., Ponti, G., Beckmann, V., \& Lubinski, P. 2010, in Proc. of The Extreme sky: Sampling the Universe above $10 \mathrm{keV}$ Conf. [arXiv: 1001.4348]

Soldi, S., Beckmann, V., Gehrels, N., de Jong, S., \& Lubiński, P. 2011a, in Narrow-Line Seyfert 1 Galaxies and their Place in the Universe [arXiv: 1105. 5993]

Soldi, S., Beckmann, V., Gehrels, N., et al. 2011b, in Proc. of the 8th INTEGRAL Workshop The Restless Gamma-ray Universe [arXiv: 1102 . 4959]

Soldi, S., Baumgartner, W., Beckmann, V., et al. 2012, in Proc. of An INTEGRAL view of the high-energy sky (the first 10 years) (INTEGRAL 2012)

Stalin, C. S., Jeyakumar, S., Coziol, R., Pawase, R. S., \& Thakur, S. S. 2011, MNRAS, 416, 225

Terashima, Y., Gallo, L. C., Inoue, H., et al. 2009, PASJ, 61, 299

Tueller, J., Baumgartner, W. H., Markwardt, C. B., et al. 2010, ApJS, 186, 378

Uttley, P. 2005, in Proc. of the AGN Variability from X-rays to Radio Waves Conf. [arXiv: 0501157]

Uttley, P., McHardy, I. M., \& Papadakis, I. E. 2002, MNRAS, 332, 231

Vasudevan, R. V., \& Fabian, A. C. 2007, MNRAS, 381, 1235

Vasudevan, R. V., \& Fabian, A. C. 2009, MNRAS, 392, 1124

Vasudevan, R. V., Mushotzky, R. F., Winter, L. M., \& Fabian, A. C. 2009, MNRAS, 399, 1553

Vasudevan, R. V., Fabian, A. C., Gandhi, P., Winter, L. M., \& Mushotzky, R. F. 2010, MNRAS, 402, 1081

Vasudevan, R. V., Mushotzky, R. F., \& Gandhi, P. 2013, ApJ, 770, L37

Vaughan, S., Edelson, R., Warwick, R. S., \& Uttley, P. 2003, MNRAS, 345, 1271

Wilson-Hodge, C. A., Cherry, M. L., Case, G. L., et al. 2011, ApJ, 727, L40

Winter, L. M., Mushotzky, R. F., Reynolds, C. S., \& Tueller, J. 2009, ApJ, 690, 1322

Winter, L. M., Veilleux, S., McKernan, B., \& Kallman, T. R. 2012, ApJ, 745, 107

Woo, J.-H., \& Urry, C. M. 2002, ApJ, 579, 530

Yaqoob, T. 2012, MNRAS, 423, 3360

Zdziarski, A. A., Lubiński, P., \& Smith, D. A. 1999, MNRAS, 303, L11

Zhang, Y.-H. 2011, ApJ, 726, 21

Zhou, X.-L., Zhang, S.-N., Wang, D.-X., \& Zhu, L. 2010, ApJ, 710, 16

Zoghbi, A., Fabian, A. C., Uttley, P., et al. 2010, MNRAS, 401, 2419

Pages 14 to 16 are available in the electronic edition of the journal at http://www . aanda. org 
Table 2. Variability estimator $S_{V}$, black hole mass, and bolometric luminosity for the selected Swift/BAT AGN sample.

\begin{tabular}{|c|c|c|c|c|c|c|c|}
\hline Source name & Type & $\begin{array}{c}\bar{x} \\
{\left[10^{-4} \mathrm{c} / \mathrm{s} / \mathrm{det}\right]}\end{array}$ & $\begin{array}{c}S_{V} \\
{[\%]} \\
\end{array}$ & $\begin{array}{c}S_{\mathrm{V}, 14-24 \mathrm{keV}} \\
{[\%]}\end{array}$ & $\begin{array}{c}S_{\mathrm{V}, 35-100 \mathrm{keV}} \\
{[\%]}\end{array}$ & $\begin{array}{c}\log M_{\mathrm{BH}} \\
{\left[M_{\odot}\right]} \\
\end{array}$ & $\begin{array}{c}\log L_{\text {bol }} \\
{\left[\operatorname{erg~s}^{-1}\right]} \\
\end{array}$ \\
\hline QSO B0033+595 & BLLac & 0.60 & $99 \pm 19$ & $94 \pm 16$ & $127_{-52}^{+47}$ & $\ldots$ & $\ldots$ \\
\hline NGC 235A & Sey1 & 0.74 & $14_{-7}^{+6}$ & & $10_{-6}^{+52}$ & $8.8 \pm 0.5^{a}$ & $\ldots$ \\
\hline Mrk 348 & Sey2 & 2.56 & $25 \pm 3$ & $30 \pm 4$ & $22 \pm 4$ & $7.2 \pm 0.7^{b}$ & $46.4^{f}$ \\
\hline Fairall 9 & Sey1 & 0.84 & $10_{-5}^{+4}$ & $\ldots$ & $\ldots$ & $8.9 \pm 0.5^{a}$ & $44.9^{\circ}$ \\
\hline NGC 526A & Sey 1.5 & 0.91 & $35 \pm 6$ & $43_{-10}^{+9}$ & $26 \pm 10$ & $8.0 \pm 0.5^{a}$ & $\ldots$ \\
\hline NGC 612 & NLRG & 0.76 & $27_{-7}^{+6}$ & $38_{-12}^{+10}$ & $9 \pm 5$ & $8.5 \pm 0.5^{a}$ & $\ldots$ \\
\hline ESO 297-018 & Sey2 & 1.01 & $16 \pm 4$ & $27 \pm 8$ & $\ldots$ & $9.7 \pm 0.5^{b}$ & $44.2^{j}$ \\
\hline NGC 788 & Sey2 & 1.19 & $15 \pm 4$ & $19_{-8}^{+7}$ & $\ldots$ & $7.5 \pm 0.7^{b}$ & $44.3^{f}$ \\
\hline LEDA 138501 & Sey 1 & 0.88 & $24 \pm 7$ & $17_{-9}^{+8}$ & $24_{-13}^{+12}$ & $\ldots$ & $\ldots$ \\
\hline NGC 931 & Sey 1.5 & 1.07 & $12 \pm 5$ & $\ldots$ & $\ldots$ & $7.6 \pm 0.3^{b}$ & $44.5^{f}$ \\
\hline [HB89] 0241+622 & Sey1 & 1.38 & $15_{-5}^{+4}$ & $18 \pm 8$ & $14_{-7}^{+6}$ & $\ldots$ & $\ldots$ \\
\hline NGC 1142 & Sey2 & 1.31 & $30 \pm 4$ & $31_{-9}^{+8}$ & $30_{-7}^{+6}$ & $9.4 \pm 0.5^{b}$ & $44.8^{j}$ \\
\hline NGC 1275 & $\mathrm{RG}$ & 1.65 & $15_{-5}^{+4}$ & $14 \pm 4$ & $25_{-14}^{+13}$ & $8.5 \pm 0.7^{b}$ & $45.0^{f}$ \\
\hline NGC 1365 & Sey1.8 & 1.09 & $22 \pm 4$ & $26_{-7}^{+6}$ & $10 \pm 5$ & $7.7 \pm 0.3^{b}$ & $43.8^{j}$ \\
\hline ESO 548-G081 & Sey1 & 0.68 & $36_{-9}^{+8}$ & $51_{-15}^{+16}$ & $33 \pm 14$ & $8.9 \pm 0.5^{a}$ & $44.0^{j}$ \\
\hline $3 \mathrm{C} 111.0$ & RG & 1.91 & $16_{-5}^{+4}$ & $15 \pm 6$ & $20_{-8}^{+7}$ & $9.6 \pm 0.8^{b}$ & $\ldots$ \\
\hline $3 \mathrm{C} 120$ & RG & 1.56 & $23 \pm 4$ & $23_{-7}^{+6}$ & $21_{-8}^{+7}$ & $7.83 \pm 0.04^{c}$ & $45.3^{f}$ \\
\hline UGC 03142 & Sey 1 & 0.77 & $34_{-10}^{+9}$ & $56_{-22}^{+21}$ & $\ldots$ & $\ldots$ & $\ldots$ \\
\hline 2MASX J04440903+2813003 & Sey2 & 0.81 & $91 \pm 17$ & $104_{-19}^{+20}$ & $73_{-20}^{+21}$ & $\ldots$ & $\ldots$ \\
\hline 1RXS J045205.0+493248 & Sey1 & 1.03 & $21_{-8}^{+7}$ & $20_{-11}^{+10}$ & $\ldots$ & $8.0 \pm 0.3^{d}$ & $45.0^{d}$ \\
\hline 2MASX J05054575-2351139 & Sey2 & 0.87 & $33 \pm 6$ & $28_{-9}^{+8}$ & $16 \pm 7$ & $7.5 \pm 0.5^{a}$ & $\ldots$ \\
\hline IRAS 05078+1626 & Sey 1.5 & 1.50 & $17_{-8}^{+6}$ & $3 \pm 2$ & $\ldots$ & $6.98_{-0.2}^{+0.01 e}$ & $\ldots$ \\
\hline Ark 120 & Sey 1 & 1.07 & $9_{-5}^{+8}$ & $\ldots$ & $\ldots$ & $8.18_{-0.06}^{+0.05 b}$ & $44.9^{f}$ \\
\hline ESO 362-18 & Sey 1.5 & 0.76 & $9 \pm 4$ & $19 \pm 9$ & $\ldots$ & $9.0 \pm 0.5^{a}$ & $\ldots$ \\
\hline PICTOR A & BLRG & 0.63 & $51_{-9}^{+10}$ & $33_{-13}^{+14}$ & $69_{-18}^{+18}$ & $7.6 \pm 0.5^{a}$ & $\ldots$ \\
\hline PKS 0521-36 & BLLac & 0.52 & $29+9$ & $55 \pm 17$ & $\ldots$ & $8.7 \pm 0.3^{f}$ & $\ldots$ \\
\hline PKS 0548-322 & BLLac & 0.55 & $24_{-10}^{+9}$ & $15_{-8}^{+7}$ & $30_{-14}^{+15}$ & $8.2 \pm 0.3^{f}$ & $\ldots$ \\
\hline NGC 2110 & Sey2 & 4.77 & $33 \pm 3$ & $35 \pm 4$ & $32 \pm 4$ & $8.3 \pm 0.3^{b}$ & $44.1^{f}$ \\
\hline MCG +08-11-011 & Sey 1.5 & 2.15 & $33_{-4}^{+5}$ & $32 \pm 5$ & $31 \pm 6$ & $8.1 \pm 0.6^{b}$ & $45.0^{j}$ \\
\hline 2MASX J05580206-3820043 & Sey 1 & 0.60 & $29_{-10}^{+9}$ & $35_{-12}^{+11}$ & $\ldots$ & $8.4 \pm 0.5^{a}$ & $\ldots$ \\
\hline IRAS 05589+2828 & Sey 1 & 1.14 & $33 \pm 8$ & $25 \pm 10$ & $\ldots$ & $\ldots$ & $44.7^{d}$ \\
\hline Mrk 3 & Sey2 & 1.86 & $35_{-9}^{+11}$ & $54_{-15}^{+20}$ & $29_{-5}^{+6}$ & $8.7 \pm 0.3^{b}$ & $44.5^{f}$ \\
\hline Mrk 6 & Sey 1.5 & 0.93 & $18_{-6}^{+5}$ & $\ldots$ & $12_{-7}^{+6}$ & $8.13 \pm 0.04^{c}$ & $44.3^{j}$ \\
\hline Mrk 79 & Sey1.2 & 0.75 & $6 \pm 4$ & $29_{-10}^{+9}$ & $\ldots$ & $8.4 \pm 0.5^{a}$ & $44.6^{f}$ \\
\hline 2MASS J07594181-3843560 & Sey 1.2 & 0.82 & $10 \pm 5$ & $31 \pm 12$ & $17_{-9}^{+8}$ & $8.3 \pm 0.5^{b}$ & $\ldots$ \\
\hline Mrk 1210 & Sey2 & 0.91 & $24_{-9}^{+8}$ & $9 \pm 6$ & $40 \pm 14$ & $6.5 \pm 0.7^{g}$ & $\ldots$ \\
\hline Fairall 272 & Sey2 & 0.64 & $50_{-13}^{+12}$ & $47_{-26}^{+25}$ & $36_{-13}^{+12}$ & $\ldots$ & $\ldots$ \\
\hline [HB89] 0836+710 & blazar & 0.90 & $12 \pm 5$ & $12_{-7}^{+5}$ & $22_{-8}^{+7}$ & $9.4 \pm 0.3^{h}$ & $\ldots$ \\
\hline MCG -01-24-012 & Sey2 & 0.70 & $19_{-10}^{+9}$ & $26_{-11}^{+12}$ & $\ldots$ & $7.2 \pm 0.5^{a}$ & $\ldots$ \\
\hline MCG +04-22-042 & Sey 1.2 & 0.63 & $27_{-11}^{+8}$ & $\ldots$ & $37_{-14}^{+12}$ & $8.5 \pm 0.5^{a}$ & $44.5^{d}$ \\
\hline Mrk 110 & NLS1 & 0.94 & $12 \pm 5$ & $24 \pm 9$ & $\ldots$ & $7.42_{-0.1}^{+0.09 b}$ & $44.7^{f}$ \\
\hline MCG -05-23-016 & Sey2 & 3.44 & $19 \pm 3$ & $23 \pm 4$ & $16 \pm 5$ & $6.3 \pm 0.5^{b}$ & $\ldots$ \\
\hline NGC 3081 & Sey2 & 1.28 & $19_{-7}^{+6}$ & $18_{-10}^{+9}$ & $17 \pm 9$ & $7.4 \pm 0.3^{b}$ & $\ldots$ \\
\hline NGC 3227 & Sey 1.5 & 1.81 & $27_{-6}^{+5}$ & $33_{-8}^{+7}$ & $25 \pm 6$ & $6.9 \pm 0.1^{i}$ & $43.9^{f}$ \\
\hline NGC 3281 & Sey2 & 1.37 & $18_{-5}^{+4}$ & $21_{-9}^{+8}$ & $3 \pm 2$ & $8.0 \pm 0.5^{b}$ & $44.2^{j}$ \\
\hline Mrk 421 & BLLac & 3.17 & $94_{-9}^{+10}$ & $88_{-8}^{+9}$ & $107 \pm 12$ & $8.3 \pm 0.3^{b}$ & $\ldots$ \\
\hline NGC 3516 & Sey 1.5 & 1.77 & $26 \pm 4$ & $39 \pm 5$ & $20_{-5}^{+4}$ & $7.50_{-0.06}^{+0.04 i}$ & $44.3^{f}$ \\
\hline
\end{tabular}

Notes. The variability estimator is computed in the $14-195 \mathrm{keV}\left(S_{V}\right), 14-24 \mathrm{keV}\left(S_{\mathrm{V}, 14-24 \mathrm{keV}}\right)$, and $35-100 \mathrm{keV}\left(S_{\mathrm{V}, 35-100 \mathrm{keV}}\right)$ bands. $\bar{x}$ is the average count rate in the full 14-195 keV band. The bolometric luminosities have been estimated from the fitting of the spectral energy distribution (see Sect. 3.3).

References. ${ }^{(a)}$ Winter et al. (2009); ${ }^{(b)}$ Beckmann et al. (2009) and references therein; ${ }^{(c)}$ Grier et al. (2012); ${ }^{(d)}$ Vasudevan et al. (2009); ${ }^{(e)}$ Stalin et al. (2011); ${ }^{(f)}$ Woo \& Urry (2002); ${ }^{(g)}$ Bian \& Gu (2007); ${ }^{(h)}$ Kaspi et al. (2007); ${ }^{(i)}$ Denney et al. (2010); ${ }^{(j)}$ Vasudevan et al. (2010); ${ }^{(k)}$ Nikolajuk et al. (2004); ${ }^{(l)}$ Greenhill et al. (2003); ${ }^{(m)}$ Padovani \& Rafanelli (1988); ${ }^{(n)}$ Malizia et al. (2008); ${ }^{(o)}$ Vasudevan \& Fabian (2007). 
S. Soldi et al.: Long-term variability of AGN at hard X-rays

Table 2. continued.

\begin{tabular}{|c|c|c|c|c|c|c|c|}
\hline Source name & Type & $\begin{array}{c}\bar{x} \\
{\left[10^{-4} \mathrm{c} / \mathrm{s} / \mathrm{det}\right]}\end{array}$ & $\begin{array}{c}S_{V} \\
{[\%]} \\
\end{array}$ & $\begin{array}{c}S_{\mathrm{V}, 14-24 \mathrm{keV}} \\
{[\%]}\end{array}$ & $\begin{array}{c}S_{\mathrm{V}, 35-100 \mathrm{keV}} \\
{[\%]}\end{array}$ & $\begin{array}{c}\log M_{\mathrm{BH}} \\
{\left[M_{\odot}\right]}\end{array}$ & $\begin{array}{l}\log L_{\text {bol }} \\
{\left[\operatorname{erg~s}^{-1}\right]}\end{array}$ \\
\hline NGC 3783 & Sey1 & 2.84 & $15_{-3}^{+2}$ & $20 \pm 3$ & $9 \pm 4$ & $7.47^{+0.07 b}$ & $44.4^{f}$ \\
\hline UGC 06728 & Sey 1.2 & 0.51 & $21_{-9}^{+3}$ & $15_{-9}^{+8}$ & $\ldots$ & $6.4 \pm 0.3^{d}$ & $44.3^{d}$ \\
\hline 2MASX J11454045-1827149 & Sey1 & 0.86 & $24_{-9}^{+8}$ & $25 \pm 13$ & $\ldots$ & $6.7 \pm 0.5^{a}$ & $\ldots$ \\
\hline LEDA 38038 & Sey2 & 0.85 & $24_{-9}^{+9}$ & $30_{-13}^{+12}$ & $\ldots$ & 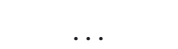 & $\ldots$ \\
\hline NGC 4151 & Sey 1.5 & 8.06 & $29_{-4}^{+3}$ & $30 \pm 4$ & $28 \pm 3$ & $7.5_{-0.6}^{+0.1 b}$ & $43.7^{f}$ \\
\hline NGC 4388 & Sey2 & 4.01 & $26 \pm 2$ & $26_{-3}^{+2}$ & $25 \pm 3$ & $7.2 \pm 0.6^{b}$ & $43.7^{j}$ \\
\hline $3 \mathrm{C} 273$ & quasar & 6.23 & $29 \pm 3$ & $29 \pm 3$ & $28 \pm 3$ & $9.81_{-0.07}^{+0.1 b}$ & $47.1^{\circ}$ \\
\hline NGC 4507 & Sey2 & 2.85 & $20 \pm 4$ & $30 \pm 5$ & $15_{-4}^{+3}$ & $7.6 \pm 0.6^{b}$ & $44.3^{j}$ \\
\hline ESO 506-G027 & Sey2 & 1.28 & $43 \pm 7$ & $43 \pm 10$ & $31 \pm 7$ & $8.6 \pm 0.5^{b}$ & $44.6^{j}$ \\
\hline NGC 4593 & Sey1 & 1.40 & $15 \pm 4$ & $17_{-7}^{+6}$ & $\ldots$ & $6.99_{-0.1}^{+0.08 b}$ & $44.1^{f}$ \\
\hline WKK 1263 & Sey1.5 & 0.72 & $36_{-9}^{+8}$ & $54 \pm 19$ & $40_{-15}^{+14}$ & $8.0 \pm 0.5^{-0.1}$ & $44.3^{d}$ \\
\hline SBS $1301+540$ & Sey1 & 0.52 & $36_{-9}^{+10}$ & $\ldots$ & $19_{-10}^{+9}$ & $7.5 \pm 0.5^{a}$ & $44.9^{d}$ \\
\hline NGC 4945 & Sey2 & 3.48 & $34 \pm 4$ & $48 \pm 10$ & $28 \pm 4$ & $6.2 \pm 0.3^{b}$ & $\ldots$ \\
\hline ESO 323-077 & Sey1.2 & 0.73 & $19_{-9}^{+8}$ & $42_{-21}^{+20}$ & $\ldots$ & $7.4 \pm 0.6^{b}$ & $\ldots$ \\
\hline Cen A & RG & 20.40 & $39 \pm 4$ & $37 \pm 4$ & $39 \pm 4$ & $8.0 \pm 0.6^{b}$ & $\ldots$ \\
\hline MCG -06-30-015 & Sey 1.2 & 1.26 & $13_{-7}^{+6}$ & $21_{-9}^{+8}$ & $\ldots$ & $6.7_{-02}^{+0.1 b}$ & $44.1^{d}$ \\
\hline NGC 5252 & Sey2 & 1.65 & $59_{-7}^{+1}$ & $61_{-9}^{+10}$ & $54 \pm 8$ & $9.03_{-0.02}^{-0.2} b$ & $45.4^{f}$ \\
\hline $4 \mathrm{U} 1344-60$ & Sey 1.5 & 1.67 & $14 \pm 4$ & $23 \pm 7$ & $7_{-5}^{+4}$ & $7.4 \pm 0.1^{j}$ & $44.1^{j}$ \\
\hline IC 4329A & Sey 1.2 & 4.82 & $17 \pm 3$ & $31 \pm 5$ & $9_{-4}^{+3}$ & $8.1 \pm 0.2^{k}$ & $44.8^{f}$ \\
\hline Mrk 279 & Sey 1.5 & 0.63 & $36_{-7}^{+6}$ & $37_{-13}^{+12}$ & $35_{-12}^{+11}$ & $8.6 \pm 0.5^{a}$ & $45.0^{\circ}$ \\
\hline Circinus Galaxy & Sey2 & 4.54 & $11 \pm 1$ & $17^{-13} 2$ & $\ldots$ & $6.3 \pm 0.1^{l}$ & $\ldots$ \\
\hline NGC 5506 & NLS1 & 4.12 & $15_{-4}^{+3}$ & $25_{-5}^{+4}$ & $8 \pm 4$ & $6.7 \pm 0.7^{b}$ & $44.1^{j}$ \\
\hline NGC 5548 & Sey 1.5 & 1.19 & $31_{-4}^{+5}$ & $19_{-8}^{+5}$ & $24_{-7}^{+6}$ & $7.82 \pm 0.02^{b}$ & $44.8^{f}$ \\
\hline ESO 511-G030 & Sey1 & 0.76 & $33 \pm 11$ & $53_{-16}^{+8}$ & $\ldots$ & $8.7 \pm 0.5^{b}$ & $44.4^{j}$ \\
\hline NGC 5728 & Sey2 & 1.35 & $27_{-7}^{+6}$ & $39_{-24}^{+15}$ & $19_{-9}^{+8}$ & $8.5 \pm 0.5^{a}$ & $43.7^{j}$ \\
\hline Mrk 841 & Sey1 & 0.64 & $21 \pm 10$ & $25_{-13}^{+12}$ & $\ldots$ & $8.5 \pm 0.7^{b}$ & $45.8^{f}$ \\
\hline PKS 1510-08 & quasar & 0.83 & $41_{-12}^{+10}$ & $89_{-36}^{+17}$ & $27_{-12}^{+11}$ & $8.7 \pm 0.5^{f}$ & $46.4^{f}$ \\
\hline VII Zw 653 & Sey 1.2 & 0.87 & $21_{-8}^{+7}$ & $32_{-11}^{+10}$ & $24_{-16}^{+12}$ & $\ldots$ & $\ldots$ \\
\hline Mrk 1498 & Sey 1.9 & 0.74 & $5 \pm 3$ & $\ldots$ & $\ldots$ & $8.6 \pm 0.5^{a}$ & $45.3^{j}$ \\
\hline 2MASX J16481523-3035037 & Sey1 & 0.77 & $47 \pm 8$ & $54_{-20}^{+16}$ & $42_{-16}^{+15}$ & $\ldots$ & $\ldots$ \\
\hline NGC 6240 & Sey2 & 1.06 & $22_{-7}^{+6}$ & $54_{-18}^{+17}$ & $4 \pm 3$ & $\ldots$ & $\ldots$ \\
\hline Mrk 501 & BLLac & 0.89 & $61 \pm 8$ & $64 \pm 9$ & $62_{-13}^{+12}$ & $9.2 \pm 0.3^{b}$ & $\ldots$ \\
\hline 1RXS J165605.6-520345 & Sey 1.2 & 0.69 & $21 \pm 10$ & $26_{-16}^{+15}$ & $21_{-12}^{+10}$ & $7.9 \pm 0.5^{b}$ & $\ldots$ \\
\hline 2MASS J16561677-3302127 & blazar & 0.87 & $41 \pm 7$ & $44_{-22}^{+10}$ & $35_{-9}^{+8}$ & $\ldots$ & $\ldots$ \\
\hline NGC 6300 & Sey2 & 1.65 & $17 \pm 3$ & $18_{-6}^{+5}$ & $8_{-5}^{+4}$ & $5.5 \pm 0.4^{b}$ & $42.9^{j}$ \\
\hline AX J1737.4-2907 & Sey1 & 1.96 & $15_{-4}^{+3}$ & $33 \pm 7$ & $\ldots$ & $8.9 \pm 0.7^{b}$ & $\ldots$ \\
\hline IC 4709 & Sey2 & 0.66 & $22_{-11}^{+10}$ & $34_{-19}^{+18}$ & $\ldots$ & $\ldots$ & $\ldots$ \\
\hline PKS 1830-21 & FSRQ & 1.04 & $18_{-8}^{+6}$ & $38_{-21}^{+19}$ & $\ldots$ & $\ldots$ & $\ldots$ \\
\hline 3 C 382 & Sey1 & 1.47 & $11_{-4}^{+3}$ & $15_{-6}^{+5}$ & $11 \pm 5$ & $9.2 \pm 0.5^{b}$ & $45.6^{\circ}$ \\
\hline ESO 103-035 & Sey2 & 1.88 & $12 \pm 3$ & $17 \pm 5$ & $\ldots$ & $7.1 \pm 0.6^{b}$ & $44.6^{j}$ \\
\hline 3C 390.3 & BLRG & 1.69 & $17 \pm 3$ & $16 \pm 5$ & $12 \pm 5$ & $8.46_{-01}^{+0.09 b}$ & $44.9^{f}$ \\
\hline Fairall 51 & Sey1 & 0.70 & $20_{-8}^{+7}$ & $45_{-19}^{+17}$ & $\ldots$ & $7.5 \pm 0.5^{m}$ & $\ldots$ \\
\hline NGC 6814 & Sey 1.5 & 1.19 & $28_{-8}^{+7}$ & $36_{-17}^{+14}$ & $19_{-9}^{+8}$ & $7.1 \pm 0.2^{b}$ & $43.9^{f}$ \\
\hline Cygnus A & RG & 2.13 & $31_{-5}^{+4}$ & $32_{-6}^{+5}$ & $35 \pm 6$ & $9.4 \pm 0.1^{b}$ & $45.7^{j}$ \\
\hline QSO B1959+650 & BLLac & 0.69 & $35_{-10}^{+9}$ & $42 \pm 9$ & $\ldots$ & $8.1 \pm 0.3^{b}$ & $\ldots$ \\
\hline $\mathrm{MCG}+04-48-002$ & Sey2 & 1.13 & $20_{-7}^{+6}$ & $24_{-9}^{+8}$ & $\ldots$ & $\ldots$ & $\ldots$ \\
\hline $4 \mathrm{C}+74.26$ & Sey1 & 0.87 & $21_{-7}^{+6}$ & $14_{-8}^{+6}$ & $28 \pm 11$ & $9.6 \pm 0.5^{b}$ & $46.2^{f}$ \\
\hline Mrk 509 & Sey 1.2 & 1.60 & $17_{-8}^{+7}$ & $16_{-9}^{+8}$ & $12_{-7}^{+6}$ & $8.16_{-0.04}^{+0.03 b}$ & $45.0^{f}$ \\
\hline IC 5063 & Sey2 & 1.07 & $18 \pm 5$ & $38 \pm 13$ & $\ldots$ & $7.7 \pm 0.5^{a}$ & $44.5^{f}$ \\
\hline 2MASX J21140128+8204483 & Sey1 & 0.55 & $12 \pm 6$ & $36_{-16}^{+15}$ & $\ldots$ & $8.8 \pm 0.5^{b}$ & $45.5^{j}$ \\
\hline 28P 206 & RG & 3.20 & $30 \pm 4$ & $29_{-4}^{+3}$ & $31 \pm 5$ & $\ldots$ & $\ldots$ \\
\hline SWIFT J212745.6+565636 & NLS1 & 0.73 & $20_{-8}^{+7}$ & $21 \pm 9$ & $20 \pm 11$ & $7.2 \pm 0.5^{n}$ & $\ldots$ \\
\hline $6 \mathrm{dF}$ J2132022-334254 & Sey1 & 0.72 & $40 \pm 7$ & $49_{-13}^{+12}$ & $36_{-16}^{+15}$ & $\ldots$ & $\ldots$ \\
\hline PKS 2149-306 & FSRQ & 1.07 & $26 \pm 7$ & $\ldots$ & $29 \pm 10$ & $\ldots$ & $\ldots$ \\
\hline
\end{tabular}


A\&A 563, A57 (2014)

Table 2. continued.

\begin{tabular}{|c|c|c|c|c|c|c|c|}
\hline Source name & Type & $\begin{array}{c}\bar{x} \\
{\left[10^{-4} \mathrm{c} / \mathrm{s} / \mathrm{det}\right]}\end{array}$ & $\begin{array}{c}S_{V} \\
{[\%]}\end{array}$ & $\begin{array}{c}S_{\mathrm{V}, 14-24 \mathrm{keV}} \\
{[\%]}\end{array}$ & $\begin{array}{c}S_{\mathrm{V}, 35-100 \mathrm{keV}} \\
{[\%]}\end{array}$ & $\begin{array}{c}\log M_{\mathrm{BH}} \\
{\left[M_{\odot}\right]}\end{array}$ & $\begin{array}{l}\log L_{\mathrm{bol}} \\
{\left[\operatorname{erg~s}^{-1}\right]}\end{array}$ \\
\hline NGC 7172 & Sey2 & 2.63 & $28 \pm 3$ & $33 \pm 7$ & $29 \pm 5$ & $7.7 \pm 0.6^{b}$ & $43.8^{j}$ \\
\hline NGC 7213 & Sey1.5 & 0.68 & $23_{-10}^{+9}$ & $\ldots$ & $\ldots$ & $8.6 \pm 0.5^{a}$ & $44.3^{f}$ \\
\hline NGC 7314 & Sey1 & 0.80 & $21_{-10}^{+9}$ & $\ldots$ & $\ldots$ & $6.0 \pm 0.5^{b}$ & $43.0^{j}$ \\
\hline $3 \mathrm{C} 454.3$ & FSRQ & 1.74 & $59 \pm 8$ & $60_{-12}^{+11}$ & $56 \pm 9$ & $9.2 \pm 0.7^{b}$ & $47.3^{f}$ \\
\hline MR 2251-178 & Sey1 & 1.67 & $10_{-5}^{+4}$ & $11_{-6}^{+5}$ & $1 \pm 1$ & $<6.9^{b}$ & $45.8^{\circ}$ \\
\hline NGC 7469 & Sey1 & 1.10 & $23 \pm 6$ & $22_{-10}^{+6}$ & $20_{-11}^{+10}$ & $7.09 \pm 0.05^{b}$ & $45.3^{f}$ \\
\hline Mrk 926 & Sey1.5 & 1.81 & $27_{-5}^{+4}$ & $26 \pm 7$ & $21_{-6}^{+5}$ & $7.1 \pm 0.6^{b}$ & $\ldots$ \\
\hline NGC 7582 & Sey2 & 1.21 & $23 \pm 5$ & $24_{-8}^{+7}$ & $12^{-6}{ }^{-6}$ & $8.3 \pm 0.5^{a}$ & $43.3^{j}$ \\
\hline NGC 7603 & Sey 1.5 & 0.82 & $11_{-7}^{+6}$ & $\ldots$ & $\ldots$ & $8.1 \pm 0.3^{b}$ & $44.7^{f}$ \\
\hline
\end{tabular}

